\title{
Publishifgsing monomer vibrational wavefunctions to compute numerically exact (12D) rovibrational levels of water dimer
}

\author{
Xiao-Gang Wang* and Tucker Carrington Jr. ${ }^{\dagger}$ \\ Chemistry Department, Queen's University, \\ Kingston, Ontario KYL 3N6, Canada
}

(Dated: Dec 14 2017)

\begin{abstract}
We compute numerically exact rovibrational levels of water dimer, with 12 vibrational coordinates, on the accurate CCpol-8sf ab initio flexible monomer potential energy surface[J. Chem. Phys. 137, 014305 (2012)]. It does not have a sum-of-products or multimode form and therefore quadrature in some form must be used. To do the calculation, it is necessary to use an efficient basis set and to develop computational tools, for evaluating the matrix-vector products required to calculate the spectrum, that obviate the need to store the potential on a $12 \mathrm{D}$ quadrature grid. The basis functions we use are products of monomer vibrational wavefunctions and standard rigid-monomer basis functions (which involve products of three Wigner functions). Potential matrix-vector products are evaluated using the $\boldsymbol{F}$ matrix idea previously used to compute rovibrational levels of 5-atom and 6-atom molecules. When the coupling between inter- and intra-monomer coordinates is weak, this crude adiabatic type basis is efficient (only a few monomer vibrational wavefunctions are necessary), although the calculation of matrix elements is straightforward. It is much easier to use than an adiabatic basis. The product structure of the basis is compatible with the product structure of the kinetic energy operator and this facilitates computation of matrix-vector products. Compared with the results obtained using a $[6+6] \mathrm{D}$ adiabatic approach, we find good agreement for the inter-molecular levels and larger differences for the intra-molecular water bend levels.
\end{abstract}

*Electronic address: xgwang.dalian@gmail.com

${ }^{\dagger}$ Electronic address: Tucker. Carrington@queensu.ca 


\section{Publishi.hg INTRODUCTION}

Both experimentalists, see Refs. 1-12 and papers cited therein and theorists [13-31] have studied the water dimer for decades. It is a prototypical hydrogen-bonded complex and hydrogen bonding is important in many biological and chemical processes. The water dimer itself is thought to play an important role in Earth's atmosphere. [32] It is also studied because of the importance of liquid water. Good models for bulk water are built on the potential energy surface (PES) of water dimer. [29, 33, 34] Several groups have measured high resolution spectra of the water dimer. Experimental studies are summarized in Ref. 12 and Ref. 11 reports recent inter-monomer levels of $\left(\mathrm{H}_{2} \mathrm{O}\right)_{2}$ near $500 \mathrm{~cm}^{-1}$. The water dimer PES has eight equivalent wells and the associated tunneling splittings have been measured with astonishing accuracy. There are also many calculations of the ro-vibrational spectrum of the water dimer. [14-23, 25-27] By comparing with experimental spectra, theorists are able to assess the accuracy of and refine ab initio PESs.

The first calculations were done with rigid monomers, i.e. by fixing the shape of the water monomers. [14, 17-21] This reduces the number of vibrational degrees of freedom (DOF) from 12 to 6 which is important because: 1) the size of the basis used to represent wavefunctions scales exponentially with the number of DOF and 2)most computational methods require storing in memory values of the PES on a grid whose size also scales exponentially with the number of DOF.[35-38] Rigid monomer calculations are done with a kinetic energy operator (KEO) derived by Brocks et al.[39] It is written in terms of Euler angles that specify the orientation of monomer frames with respect to a frame attached to the inter-monomer Jacobi vector. The basis functions are products of three Wigner functions, one for each of the monomers and one for overall rotation of the complex. Owing to the fact that even the rigid-monomer basis is large (for small values of $J$ it has millions of functions), it is advantageous to use an iterative eigensolver. [40-42] Similar rigid-monomer calculations have been done for other complexes. [43-52]

Tunneling splittings computed with the rigid-monomer KEO and accurate ab initio PESs agree extremely well with their experimental counterparts,[21] however, it is important to develop tools that enable one to compute the water dimer spectrum without making the rigid-monomer approximation. This is important because 1) the best possible refined water 
Publishidign er PES must be obtained from an exact calculation and 2) it is only by doing the 12D calculation that it will be possible to compute shifts of intra-monomer frequencies. The best $12 \mathrm{D}$ calculations are those of Leforestier et al. who used a [6+6]D adiabatic approach. $[25,26]$. In this adiabatic approach, a 6D "fast" Schroedinger equation is solved for the intra-monomer coordinates at each point on a grid of inter-monomer "slow" coordinates and a slow Schroedinger equation, for which the potential is obtained from the lowest eigenvalue of the fast Schroedinger equation, is solved to obtain the final energy levels. To implement this strategy, one must neglect terms in the KEO that couple slow and fast coordinates and introduce additional approximations to deal with slow coordinate dependence that is introduced into the slow KEO due to the parametric dependence of the fast wavefunctions on the slow coordinates. It is important to test the accuracy of these approximations. For water dimer they work well for the lowest energy levels, for other Van der Waals or hydrogen-bonded complexes they might not. The best way to test the approximations is to do a numerically exact calculation.

If the rigid-monomer basis has millions of functions, then it is clearly difficult to add six intra-monomer coordinates and solve the 12D problem. The most obvious intra-monomer basis functions are products of functions of each of the intra-monomer coordinates. For example, one might use $\mathbf{q}_{\mathbf{A}}=\left\{R_{1 A}, R_{2 A}, \theta_{A}\right\}$ and $\mathbf{q}_{\mathbf{B}}=\left\{R_{1 B}, R_{2 B}, \theta_{B}\right\}$, for the coordinates of monomers $\mathrm{A}$ and $\mathrm{B}$, and a basis of products of univariate functions of $R_{1 A}, R_{2 A}, \theta_{A}, R_{1 B}, R_{2 B}$, and $\theta_{B}$. The most obvious basis functions for the 12D calculation are products of the rigidmonomer basis functions and one of the univariate intra-monomer functions for each intramonomer coordinate. If 10 functions are required for each of the intra-monomer coordinates then the $12 \mathrm{D}$ basis is a factor of $10^{6}$ larger than the rigid-monomer basis. New ideas are necessary

It would be possible to extend the $[6+6]$ adiabatic approach of Leforestier. This could be done by including the terms he neglects, dealing properly with the coordinate dependence of the slow KEO, computing non-adiabatic coupling etc, but it would be costly and complicated. Instead, we use ideas similar to those that made it possible to compute ro-vibrational energy levels of methane and $\mathrm{CH}_{5}^{+}$. The key idea is to replace the adiabatic approximation with a crude-adiabatic approximation.[41, 53, 54] Solutions of an intra-monomer Schroedinger equations are used as basis functions for the full problem.[55] It is critical that it be possible 
Publishinggcompute matrix elements in this basis without storing values of the PES on a $12 \mathrm{D}$ grid.

Of course, calculations of this type are impossible without a PES. Due to the importance of water dimer, there are many high-quality potential energy surfaces. A recent review[31] provides a good summary. The most accurate rigid monomer (6D) PES is CCpol8s PES[21]. The refined SAPT-5st 6D surface[19] is also of high quality and has been studied extensively[20]. The best 12D PESs are: HBB2[28] and WHBB[29], developed by Bowman and co-workers; MB-pol[30] developed by Paesani and co-workers; and CCpol-8sf[26, 31] developed by Szalewicz and co-workers. In this paper we use the CCpol-8sf PES. The primary reason for using CCpol-8sf PES is that Leforestier and co-workers reported detailed results obtained by applying the $[6+6] \mathrm{D}$ adiabatic approach on that PES. [26] A secondary reason is that we find the CCpol-8sf PES has no holes within the-region covered by our extensive quadrature grids, but that MB-pol PES[30] does. The $[6+6] \mathrm{D}$ results also agree well with experiment.[26] We note that $[6+6] \mathrm{D}$ method has also been applied to the HBB2[27] and MB-pol[30] surfaces.

In this paper, we extend the method of Ref. 55 and use it to compute ro-vibrational levels of water dimer. First, we compute inter-monomer levels, for which we expect the $[6+6]$ adiabatic method of Leforestier to be accurate. Second, we compute some low-lying intra-molecular levels to obtain frequency shifts. Frequency shifts obtained from the $[6+6]$ adiabatic method have larger errors than the inter-monomer levels.

\section{KINETIC ENERGY OPERATOR}

We use the KEO of Brocks et al. [39] The coordinates are,

$$
\begin{aligned}
& \text { rotation : } \alpha, \beta \\
& \text { vibration : } \underbrace{\left(R_{1 A}, R_{2 A}, \theta_{A}\right)}_{\mathbf{q}_{\mathbf{A}}}, \underbrace{\left(R_{1 B}, R_{2 B}, \theta_{B}\right)}_{\mathbf{q}_{\mathbf{B}}}, \underbrace{\left(r_{0}, \alpha_{A}, \beta_{A}, \gamma_{A}, \alpha_{B}, \beta_{B}, \gamma_{B}\right)}_{\mathbf{Q}} .
\end{aligned}
$$

See Fig. 1. The dashed black $(x, y, z)$ axes (the $y$ axis not shown) are those of a dimer-fixed (DF) frame obtained by rotating the space-fixed (SF) frame by two Euler angles $(\alpha, \beta)$. The blue $z$ axes are those of the monomer-fixed(MF) frames. We use the same MF frame for $\mathrm{H}_{2} \mathrm{O}$ as Leforestier et al. [26] The MF $z$ axis is along the bisector of the Radau angle and it points away from the $\mathrm{H}$ atoms. The $x$ axis is in the plane of $\mathrm{H}_{2} \mathrm{O}$ and the $y$ axis (not shown) 
Publishiing erpendicular to the plane of $\mathrm{H}_{2} \mathrm{O} .\left(\alpha_{A}, \beta_{A}, \gamma_{A}\right) /\left(\alpha_{B}, \beta_{B}, \gamma_{B}\right)$ specify the orientation of the MF frame of monomer $\mathrm{A} / \mathrm{B}$ with respect to the $\mathrm{DF}$ frame. Note that the rotation of the dimer is described by the two Euler angles that specify the DF frame. The DF frame is equivalent to the $\mathrm{E} 2$ frame of Gatti and co-workers. [56] In the rest of the paper, $\left(\mathbf{q}_{\mathbf{A}}, \mathbf{q}_{\mathbf{B}}\right)$ refer to vibrational coordinates of the monomers $\mathrm{A}$ and $\mathrm{B}$, $\mathbf{q}$ refers to the vibrational coordinates of both monomers and $\mathbf{Q}$ refers to the inter-monomer coordinates. The PES depends on only six of the seven $\mathbf{Q}$ coordinates. $\alpha_{A}$ can be considered a third rotational coordinate, but $(\alpha, \beta)$ by themselves do define a body-fixed frame.

The general KEO of Brocks et al.[39] is

$$
T=T^{A}\left(\mathbf{q}_{\mathbf{A}} ; \mathbf{p}_{\mathbf{A}}, \mathbf{j}_{\mathbf{A}}^{\mathrm{MF}}\right)+T^{B}\left(\mathbf{q}_{\mathbf{B}} ; \mathbf{p}_{\mathbf{B}}, \mathbf{j}_{\mathbf{B}}^{\mathrm{MF}}\right)+T_{i n t}\left(\mathbf{Q} ; \mathbf{p}_{\mathbf{r}_{\mathbf{0}}}, \mathbf{j}_{\mathbf{A}}, \mathbf{j}_{\mathbf{B}}, \mathbf{J}\right)
$$

where

$$
T^{A}=\frac{1}{2}\left(\begin{array}{ll}
\mathbf{p}_{\mathbf{A}}^{\dagger} & \mathbf{j}_{\mathbf{A}}^{\mathrm{MF} \dagger}
\end{array}\right)\left(\begin{array}{cc}
\mathbf{G}\left(\mathbf{q}_{\mathbf{A}}\right) & \sigma\left(\mathbf{q}_{\mathbf{A}}\right) \\
\sigma^{\mathbf{t}}\left(\mathbf{q}_{\mathbf{A}}\right) & \Gamma\left(\mathbf{q}_{\mathbf{A}}\right)
\end{array}\right)\left(\begin{array}{c}
\mathbf{p}_{\mathbf{A}} \\
\mathbf{j}_{\mathbf{A}}^{\mathrm{MF}}
\end{array}\right)
$$

and

$$
T_{\text {int }}=-\frac{1}{2 \mu_{0}} \frac{\partial^{2}}{\partial r_{0}^{2}}+B_{0}\left(r_{0}\right)\left[\mathbf{J}^{2}-\cot \beta \frac{\partial}{\partial \beta}+\left(\mathbf{j}_{\mathbf{A}}+\mathbf{j}_{\mathbf{B}}\right)^{2}-2\left(\mathbf{j}_{\mathbf{A}}+\mathbf{j}_{\mathbf{B}}\right) \cdot \mathbf{J}\right] .
$$

The vibrational coordinates and momenta upon which each term depends are indicated explicitly. $T^{X}$ is a standard triatomic rovibrational $\mathrm{KEO}$ for monomer $\mathrm{X}$. It is written in terms of vibrational coordinates $\mathbf{q}_{\mathbf{x}}$ and in a MF frame whose orientation is specified by $\left(\alpha_{X}, \beta_{X}, \gamma_{X}\right)$. The matrices $\mathbf{G}\left(\mathbf{q}_{\mathbf{A}}\right)$ and $\sigma\left(\mathbf{q}_{\mathbf{A}}\right)$ depend on the choice of the vibrational coordinates. The matrices $\sigma\left(\mathbf{q}_{\mathbf{A}}\right)$ and $\boldsymbol{\Gamma}\left(\mathbf{q}_{\mathbf{A}}\right)$ depend on the choice of the MF frame. We use Radau coordinates[57] and the bisector MF frame (called $f=1$ in Refs. 26, 58). In this paper, we shall write

$$
\begin{aligned}
T^{A} & =T_{v}^{A}+T_{r}^{A}+T_{\text {Cori }}^{A} \\
T_{v}^{A} & =\frac{1}{2} \mathbf{p}_{\mathbf{A}}^{\dagger} \mathbf{G}\left(\mathbf{q}_{\mathbf{A}}\right) \mathbf{p}_{\mathbf{A}} \\
T_{r}^{A} & =\frac{1}{2} \mathbf{j}_{\mathbf{A}}^{\mathrm{MF} \dagger} \boldsymbol{\Gamma}\left(\mathbf{q}_{\mathbf{A}}\right) \mathbf{j}_{\mathbf{A}}^{\mathrm{MF}} \\
T_{\text {Cori }}^{A} & =\frac{1}{2}\left[\mathbf{j}_{\mathbf{A}}^{\mathrm{MF} \dagger} \sigma^{\mathbf{t}}\left(\mathbf{q}_{\mathbf{A}}\right) \mathbf{p}_{\mathbf{A}}+\mathbf{p}_{\mathbf{A}}^{\dagger} \sigma\left(\mathbf{q}_{\mathbf{A}}\right) \mathbf{j}_{\mathbf{A}}^{\mathrm{MF}}\right]
\end{aligned}
$$

$T_{\text {int }}$ includes all the inter-molecular terms and it depends on $\mathbf{Q}$.

Components of all the angular momentum operators in $T_{\text {int }}$ are projected onto the DF frame. $\mathbf{J}$ is the total angular momentum, and $\mathbf{j}=\mathbf{j}_{A}+\mathbf{j}_{B}$ the sum of the angular momenta 
Publishipfornonomers $\mathrm{A}$ and $\mathrm{B}$. In contrast, components of angular momentum operators in $T^{A}$ and $T^{B}$ are projected onto the MF frames. Therefore, $\mathbf{j}_{X}$ in $T_{i n t}$ and $\mathbf{j}_{\mathbf{X}}^{\mathrm{MF}}$ in $T^{X}$ are monomer angular momenta projected onto different frames and have different analytical expressions in terms of $\left(\alpha_{X}, \beta_{X}, \gamma_{X}\right)[59]$. They are both functions of $\mathbf{Q}$. The KEO is described in more detail in many papers, e.g. Ref. 26, 39, 55.

\section{BASIS FUNCTIONS}

Each of our basis functions is

$$
\left[\phi_{v}\left(\mathbf{q}_{\mathbf{A}}, \mathbf{q}_{\mathbf{B}}\right)\right]\left[f_{\alpha_{0}}\left(r_{0}\right) u_{j_{A} k_{A} m_{A} ; j_{B} k_{B} m_{B} ; K}^{J M P}\left(\alpha_{A}, \beta_{A}, \gamma_{A}, \alpha_{B}, \beta_{B}, \gamma_{B} ; \alpha, \beta\right)\right],
$$

where $\phi_{v}\left(\mathbf{q}_{\mathbf{A}}, \mathbf{q}_{\mathbf{B}}\right)=\phi_{v_{A}}^{A}\left(\mathbf{q}_{\mathbf{A}}\right) \phi_{v_{B}}^{B}\left(\mathbf{q}_{\mathbf{B}}\right)$ is a product of monomer vibrational wavefunctions (MVW) for the two monomers, $f_{\alpha_{0}}\left(r_{0}\right)$ is a discrete variable representation (DVR) function for the inter-monomer separation distance $r_{0}$ and $u_{j_{A} k_{A} m_{A} ; j_{B} k_{B} m_{B} ; K}^{J M P}$ is a rigid-monomer parity-adapted rovibrational basis function defined in Eq. (A6) in appendix A. There is a constraint on the basis function indices: $K \equiv m_{A}+m_{B}$. Due to this constraint, none of the matrix elements are singular[39, 55], and matrix elements of the operators $J^{2}-\cot \beta \frac{\partial}{\partial \beta}$ and $J_{ \pm}$act on $\left|j_{A} k_{A} m_{A} ; j_{B} k_{B} m_{B} ; J K\right\rangle$ basis functions (see Appendix A) as if they were standard body-fixed angular momentum operators. Because of the constraint, either $m_{A}$ or $m_{B}$ is not an independent label. We have chosen $m_{A}$ and $K$ to be the independent labels. In this paper, a composite label $L \equiv\left(\alpha_{0}, j_{A} k_{A} m_{A} ; j_{B} k_{B} m_{B} ; J K M ; P\right)$ is often used. $|L\rangle$ will represent the second factor in the basis function of Eq. (6).

The MVW $\phi_{v_{X}}^{X}\left(\mathbf{q X}_{\mathbf{X}}\right)$ is the solution of a 3D Hamiltonian for monomer $\mathrm{X}(X=A, B)$,

$$
H^{X}=T_{v}^{X}+V_{\mathrm{ref}}^{X}\left(\mathbf{q}_{\mathbf{x}}\right)
$$

with the energy $\mathcal{E}_{v_{X}}^{X}$

$$
H^{X} \phi_{v_{X}}^{X}(\mathbf{q} \mathbf{x})=\mathcal{E}_{v_{X}}^{X} \phi_{v_{X}}^{X}(\mathbf{q} \mathbf{x})
$$

The 3D reference Hamiltonian $H^{X}$ is specified by choosing a 3D reference potential for monomer X. One might choose $V_{\text {ref }}^{A}\left(\mathbf{q}_{\mathbf{A}}\right)=V\left(\mathbf{q}_{\mathbf{A}}, \mathbf{q}_{\mathbf{B}}^{\mathbf{e q}}, \mathbf{Q}^{\mathbf{e q}}\right)$, where $\mathbf{q}_{\mathbf{B}}^{\text {eq }}$ and $\mathbf{Q}^{\mathbf{e q}}$ are equilibrium values. Another option is $V_{\text {ref }}^{A}\left(\mathbf{q}_{\mathbf{A}}\right)=V\left(\mathbf{q}_{\mathbf{A}}, \mathbf{q}_{\mathbf{B}}\left(\mathbf{q}_{\mathbf{A}}\right), \mathbf{Q}\left(\mathbf{q}_{\mathbf{A}}\right)\right)$, where $\mathbf{q}_{\mathbf{B}}\left(\mathbf{q}_{\mathbf{A}}\right)$ and $\mathbf{Q}\left(\mathbf{q}_{\mathbf{A}}\right)$ are values that minimize the potential. In this paper, $V_{\mathrm{ref}}^{X}\left(\mathbf{q}_{\mathbf{x}}\right)$ is the potential of an isolated monomer. 
Publishing The key difference between our basis and the basis of Leforestier et al. [26] is that our $|v\rangle$ do not depend on $\mathbf{Q}$. Leforestier et al. use only a single fast basis function, but it is different for different $\mathbf{Q}$. The most obvious disadvantage of the adiabatic approach is the need to compute solutions to the fast Schroedinger equation for many values of $\mathbf{Q}$. For water dimer, one needs about a million values of $\mathbf{Q}$. These are the quadrature points used to solve the slow Schroedinger equation. In principle, it is possible to use more than one fast adiabatic basis function to compute exact $12 \mathrm{D}$ wavefunctions. If non-adiabatic coupling is weak then one expects that fewer adiabatic basis functions than MVW-will be required. However: 1) It is difficult to deal with terms in the KEO that have both slow and fast momentum operators (Leforestier et al. neglect them). They are larger if an axis of the MF frame is not along the bisector. [25] 2) Non-adiabatic coupling matrix elements are costly to compute. 3) The $\mathbf{Q}$ dependence of the fast wavefunctions introduces additional $\mathbf{Q}$ dependence into the KEO of the slow Schroedinger equation, which increases the cost of solving it. More precisely, in the adiabatic treatment, matrix elements, $\overline{\boldsymbol{\Gamma}}_{\mathbf{v}, \mathbf{v}}^{\mathbf{A}}(\mathbf{Q})=\left\langle\phi_{v}\left(\mathbf{q}_{\mathbf{A}}, \mathbf{q}_{\mathbf{B}} ; \mathbf{Q}\right)\left|\mathbf{\Gamma}\left(\mathbf{q}_{\mathbf{A}}\right)\right| \phi_{v}\left(\mathbf{q}_{\mathbf{A}}, \mathbf{q}_{\mathbf{B}} ; \mathbf{Q}\right)\right\rangle$, obtained after integrating over the fast coordinates are dependent on the slow coordinates Q. The corresponding term in the slow kinetic matrix is

$$
\frac{1}{2}\left\langle L\left|\mathbf{j}_{\mathbf{A}}^{\mathrm{MF} \dagger} \overline{\boldsymbol{\Gamma}}_{\mathbf{v}, \mathbf{v}}^{\mathbf{A}}(\mathbf{Q}) \mathbf{j}_{\mathbf{A}}^{\mathrm{MF}}\right| L\right\rangle
$$

$\overline{\boldsymbol{\Gamma}}_{\mathbf{v}, \mathbf{v}}^{\mathbf{A}}(\mathbf{Q})$ is like a coordinate-dependent $\boldsymbol{G}$ matrix element[60] and to deal with it one must use quadrature. In our method, $\left\langle\phi_{v^{\prime}}\left(\mathbf{q}_{\mathbf{A}}\right)\left|\boldsymbol{\Gamma}\left(\mathbf{q}_{\mathbf{A}}\right)\right| \phi_{v}\left(\mathbf{q}_{\mathbf{A}}\right)\right\rangle$ is a constant. Despite these disadvantages, for the purpose of calculating the lowest levels, the adiabatic approach is excellent. However, as the density of states increases, coupling becomes more important and the approximations of Leforestier et al. become more severe.

Although a single MVW is not sufficient, matrix elements in the $|v L\rangle$ basis are easy to compute. To derive equations for the matrix elements, we carve monomer Hamiltonians $H^{A}$ and $H^{B}$ out of the full Hamiltonian,

$$
H=H^{A}+H^{B}+\Delta V\left(\mathbf{q}_{\mathbf{A}}, \mathbf{q}_{\mathbf{B}}, \mathbf{Q}\right)+T_{\mathrm{int}}+T_{r}^{A}+T_{\text {Cori }}^{A}+T_{r}^{B}+T_{\text {Cori }}^{B}
$$

where

$$
\Delta V\left(\mathbf{q}_{\mathbf{A}}, \mathbf{q}_{\mathbf{B}}, \mathbf{Q}\right)=V\left(\mathbf{q}_{\mathbf{A}}, \mathbf{q}_{\mathbf{B}}, \mathbf{Q}\right)-V_{\mathrm{ref}}^{A}\left(\mathbf{q}_{\mathbf{A}}\right)-V_{\mathrm{ref}}^{B}\left(\mathbf{q}_{\mathbf{B}}\right)
$$

In the $|v\rangle$ basis, matrix elements of $H^{A}+H^{B}$ are trivial and one finds, 


$$
\begin{aligned}
\left\langle v^{\prime}|H| v\right\rangle & =\frac{1}{2} \mathbf{j}_{\mathbf{A}}^{\mathrm{MF} \dagger}\left\langle v^{\prime}\left|\boldsymbol{\Gamma}^{\mathbf{A}}\right| v\right\rangle \mathbf{j}_{\mathbf{A}}^{\mathrm{MF}}+\frac{1}{2} \mathbf{j}_{\mathbf{A}}^{\mathrm{MF} \dagger}\left\langle v^{\prime}\left|\sigma_{\mathbf{A}}^{\mathbf{t}} \mathbf{p}_{\mathbf{A}}\right| v\right\rangle+\frac{1}{2}\left\langle v^{\prime}\left|\mathbf{p}_{\mathbf{A}}^{\dagger} \sigma_{\mathbf{A}}\right| v\right\rangle \mathbf{j}_{\mathbf{A}}^{\mathrm{MF}} \\
& +\frac{1}{2} \mathbf{j}_{\mathbf{B}}^{\mathrm{MF} \dagger}\left\langle v^{\prime}\left|\boldsymbol{\Gamma}^{\mathbf{B}}\right| v\right\rangle \mathbf{j}_{\mathbf{B}}^{\mathrm{MF}}+\frac{1}{2} \mathbf{j}_{\mathbf{B}}^{\mathrm{MF} \dagger}\left\langle v^{\prime}\left|\sigma_{\mathbf{B}}^{\mathbf{t}} \mathbf{p}_{\mathbf{B}}\right| v\right\rangle+\frac{1}{2}\left\langle v^{\prime}\left|\mathbf{p}_{\mathbf{B}}^{\dagger} \sigma_{\mathbf{B}}\right| v\right\rangle \mathbf{j}_{\mathbf{B}}^{\mathrm{MF}} \\
& +T_{\mathrm{int}} \delta_{v^{\prime}, v}+\left\langle v^{\prime}\left|\Delta V\left(\mathbf{q}_{\mathbf{A}}, \mathbf{q}_{\mathbf{B}}, \mathbf{Q}\right)\right| v\right\rangle+\left(\mathcal{E}_{v_{A}}^{A}+\mathcal{E}_{v_{B}}^{B}\right) \delta_{v^{\prime}, v} .
\end{aligned}
$$

Each of the $\langle\cdots\rangle$ matrix elements on the right hand side is an operator that depends on $\mathbf{Q}, \alpha, \beta$. Elements of the full Hamiltonian matrix are $\left\langle L^{\prime}\left|\left\langle v^{\prime}|H| v\right\rangle\right| L\right\rangle$.

With the exception of the potential, all of the terms in the Hamiltonian are products of operators that depend on $\mathbf{Q}$ and/or $\alpha, \beta$ and operators that depend on $\mathbf{q}$ and therefore their matrix elements are themselves products, e.g., $\left\langle L^{\prime}\left|\left(\left(j_{A}^{\mathrm{MF} \dagger}\right)_{x}\right)^{2}\right| L\right\rangle\left\langle v^{\prime}\left|\boldsymbol{\Gamma}_{x x}^{\mathbf{A}}\right| v\right\rangle$. The $|v L\rangle$ product basis and the factorizable operator have compatible structure. $T_{\text {int }}$ is independent of $\mathbf{q}$ and hence diagonal in the $|v\rangle$ basis. Tennyson and co-workers[22, 23] use a single $|v\rangle$. In that case, one can pre-compute and store the $\left\langle v^{\prime}|\cdots| v\right\rangle$ matrix elements and the kinetic energy matrix elements are the same as those required for a rigid-monomer calculation. The most difficult term is $\Delta V$. In our basis, its matrix elements are, $\Delta \mathbf{V}_{v^{\prime} L^{\prime}, v L}=\left\langle v^{\prime} L^{\prime}|\Delta V| v L\right\rangle$. To evaluate the corresponding matrix-vector products we shall use the $\boldsymbol{F}$ matrix idea. [53, 54] It is crucial because it obviates the need to compute $\Delta \mathbf{V}$ and reduces the cost of the matrixvector products. The $\boldsymbol{F}$ matrix is discussed in detail in sections V and VI. Overall, although it is necessary to use more than one MVW, it is certainly a wise choice because matrix elements in the $|v\rangle|L\rangle$ basis are easily computed.

\section{SYMMETRY OF THE BASIS FUNCTIONS}

It is useful to exploit symmetry for two reasons. First, it can be used to reduce the number of matrix-vector products required to compute converged energy levels and the size of the vectors one must store. Second, having symmetry labels helps us to understand the energy levels. The lowest energy levels of water dimer are computed with a symmetry adapted Lanczos (SAL) method without re-orthogonalization of the Lanczos vectors. [61, 62] Some higher levels are computed with ARPACK. [63] In the SAL calculation, the size of the vectors is not reduced by exploiting symmetry, but the number of required matrix-vector products is reduced. In the ARPACK calculation, symmetry is used to decrease the number 
Publishipfornatrix-vector products and the size of the vectors. Symmetry is used differently in these two calculations, but in both cases we use projection operators.

The projection operators are linear combinations of group operations. The full PI group of $\left(\mathrm{H}_{2} \mathrm{O}\right)_{2}$ is $G_{16}=\left\{E, P_{a b}\right\} \otimes G_{8}$, where $G_{8}=G_{4} \otimes G_{2}, G_{4}=\{E,(12)\} \otimes\{E,(34)\}$, and $G_{2}=\left\{E, E^{*}\right\}$. To use the projection operators, we must determine how the group operations affect the parity-adapted basis functions, $\left|v_{A} v_{B}\right\rangle u_{j_{A} k}^{J M P} m_{A} ; j_{B} k_{B} m_{B ;} K$. To do this, we first determine the effect of $E^{*},(12),(34)$ and $P_{a b}$ on the non-parity-adapted rigid-monomer basis functions. It is summarized in Eqs. A2 to A5 (in-Appendix A). From them, we them derive the effect of the same operations on the parity-adapted rigid-monomer basis functions. It is summarized in Eqs. A11 to A14. The effect of $P_{a b}$ on the rigid-monomer parity-adapted basis functions is complicated by the constraint on the basis indices. See Eq. (A14) of appendix A. The effect of the operations on the $12 \mathrm{D}$ basis functions is more complicated because $\left|v_{A} v_{B}\right\rangle$ is also affected by the symmetry operations. Take (12) as an example. The monomer A vibrational wavefunction is either symmetric or anti-symmetric when acted upon by (12)

$$
(12)\left|v_{A} v_{B}\right\rangle=s\left(v_{A}\right)\left|v_{A} v_{B}\right\rangle
$$

where the prefactor $s\left(v_{A}\right)=1$ or -1. Using Eq. (13), Eq. (A11), Eq. (A12), Eq. (A13) and Eq. (A14), the effect of symmetry operations on the $12 \mathrm{D}$ parity-adapted basis functions is determined,

$$
\begin{aligned}
E^{*}\left|v_{A} v_{B}\right\rangle u_{j_{A} k_{A} m_{A} ; j_{B} k_{B} m_{B} ; K}^{J M P} & =(-1)^{P}\left|v_{A} v_{B}\right\rangle u_{j_{A} k_{A} m_{A} ; j_{B} k_{B} m_{B} ; K}^{J M P} \\
(12)\left|v_{A} v_{B}\right\rangle u_{j_{A} k_{A} m_{A} ; j_{B} k_{B} m_{B} ; K}^{J M P} & =s\left(v_{A}\right)(-1)^{k_{A}}\left|v_{A} v_{B}\right\rangle u_{j_{A} k_{A} m_{A} ; j_{B} k_{B} m_{B} ; K}^{J M P} \\
(34)\left|v_{A} v_{B}\right\rangle u_{j_{A} k_{A} m_{A} ; j_{B} k_{B} m_{B} ; K}^{J M P} & =s\left(v_{B}\right)(-1)^{k_{B}}\left|v_{A} v_{B}\right\rangle u_{j_{A} k_{A} m_{A} ; j_{B} k_{B} m_{B} ; K}^{J M P}
\end{aligned}
$$

and

$$
\begin{aligned}
& P_{a b}\left|v_{A} v_{B}\right\rangle u_{j_{A} k_{A} m_{A} ; j_{B} k_{B} m_{B} ; K}^{J M P}=(-1)^{j_{A}+k_{A}+j_{B}+k_{B}+P}\left|v_{B} v_{A}\right\rangle u_{j_{B} \bar{k}_{B} m_{B} ; j_{A} \bar{k}_{A} m_{A} ; K}^{J M P} \\
& \text { ( if } K>0 \text { or } m_{A}=K=0 \text { and } k_{B}<0 \text { ) } \\
& =(-1)^{J+j_{A}+j_{B}}\left|v_{B} v_{A}\right\rangle u_{j_{B} k_{B} \bar{m}_{B} ; j_{A} k_{A} \bar{m}_{A} ; 0}^{J M P} \\
& \text { ( otherwise). }
\end{aligned}
$$

The effect of the symmetry operations is more complicated in the parity-adapted case. However, it is worth the effort because it reduces the Lanczos vector length by a factor of two. 
Publishing] Projection operators for each operation in $G_{16}$ are made using the character table given by Dyke[13] (but we, as explained in Ref. 64, use A/B not for $(a b)(1324)$ but for $(a b)(13)(24)$, which is equivalent to $P_{a b}$, used in this paper). For projection operators for 2D representations, $P^{E_{a}}$ and $P^{E_{b}}$, one needs $2 \times 2$ matrices representing the operators and we use those from Table C.10 of the book by Cornwall[65]. The $G_{8}$ projection operators are defined in Table I. They are the same as those given in Table 9 of Ref. 20, except we give $P^{E_{b}}$ explicitly; it is the same as $P_{a b} P^{E_{a}}$, given in Ref. 20 .

In the SAL calculation we use the parity-adapted basis of Appendix A. Separate calculations are done for each parity using the $G_{16}$ projection operators. The size of the Lanczos vectors is the number of parity-adapted basis functions. For each parity, the $A_{1}, A_{2}, B_{1}, B_{2}$, and $E$ state are computed simultaneously.

To calculate frequency shifts we need levels (and their corresponding wavefunctions) high enough in the spectrum that it is costly to compute them without re-orthogonalization because of the large number of eigenvalue copies. Instead, we use ARPACK, which is a restarted Arnoldi method.[63] To do so, we must store many (more than the number of desired levels) vectors in memory. For the ARPACK calculations we therefore use a $G_{16}$ symmetry-adapted basis. The Arnoldi vectors are represented in a symmetry-adapted basis and energy levels are computed separately for each symmetry. For example, the $A_{1}^{+} G_{16}$ symmetry-adapted basis is about 8 times smaller than the parity-adapted basis used in the SAL calculation. This savings of a factor of 8 is significant because thousands of vectors must be stored when using ARPACK. We typically store 10-20\% more Arnoldi vectors than the number of levels we compute. Owing to the fact that the symmetry-adapted basis functions are linear combinations of the parity-adapted functions, matrix-vector products in the symmetry-adapted basis would be costly. Rather than doing matrix-vector products directly in the symmetry-adapted basis, we transform Arnoldi vectors from the symmetryadapted basis to the parity-adapted basis, which we call unpacking, evaluate the matrixvector product in the parity-adapted basis and then transform back to the symmetry-adapted basis, which we call packing. The matrix-vector product in the parity-adapted basis is efficient, due to its product structure. To do the packing and unpacking, one needs to establish a mapping between elements of a $G_{16}$ symmetry-adapted (SA) vector and elements of a parity-adapted vector. This is easily done once one has expressions for the $G_{16}$ basis 


\section{Publishifngictions.}

The $G_{16}$ basis functions can of course be obtained by applying $G_{16}$ projection operators to the PA basis functions. It is useful to think about doing this in steps. In the first step, $G_{8}$ functions are obtained by applying the $G_{4}$ projection operators of Table II to the PA functions. The labels of the $G_{8}$ basis functions are: $A_{1}^{ \pm}+B_{1}^{ \pm}, A_{2}^{ \pm}+B_{2}^{ \pm}, E_{a}^{ \pm}$, and $E_{b}^{ \pm}$. One finds that each PA basis function is also a $G_{8}$ function. For example, the $A_{1}^{ \pm}+B_{1}^{ \pm} G_{8}$ functions are the PA functions in the top set of four functions in Table II. For non-degenerate basis functions, there is a second step in which $\left(E \pm P_{a b}\right)$ is applied to the $G_{8}$ functions to find $G_{16}$ functions.

In the rigid-monomer case, there would be only a single entry in the rightmost column of Table II for each projection operator. If the monomers are-non-rigid there are four. In the rigid monomer case, a $A_{1}+B_{1}$ function, that is symmetric under (12) and (34), must have $\left(k_{A}, k_{B}\right)=($ even, even $)$. In the non-rigid case, there are (even, even), (odd, odd $),($ even, odd) and (odd,even) functions, each of which is paired with different MVW, as shown in the first 4 lines of Table II.

As an example, we illustrate the process of obtaining $G_{16}$ symmetry-adapted functions by applying projection operators to a PA function,

$$
\left|v_{A}^{B} v_{B}^{B}\right\rangle \mid u_{j_{A} k_{A}^{\text {odd }} m_{A} ; j_{B} k_{B}^{\text {odd }} m_{B} ; K}^{J 00}
$$

with $K>0$ and $P=0$ where the superscript $B$ denotes a $\mathrm{H}_{2} \mathrm{O}$ MVW anti-symmetric under $\mathrm{H}-\mathrm{H}$ permutation/and in Table II the superscript $A$ denotes symmetric $\mathrm{H}_{2} \mathrm{O}$ MVWs) and the superscript odd implies that $k_{A}$ or $k_{B}$ is odd (and in Table II the superscript even implies that $k_{A}$ or $k_{B}$ is even). The PA functions of Eq. (18) are themselves $G_{8}$ symmetry-adapted functions. This is shown by applying the projection operator $P^{A_{1}+B_{1}}$ to the PA basis function and using Eq. (15) and Eq. (16). The $A_{1}^{+}+B_{1}^{+} G_{8}$ function is written,

$$
\left|v_{A}^{B} v_{B}^{B}, L, A_{1}^{+}+B_{1}^{+}\right\rangle=\left|v_{A}^{B} v_{B}^{B}\right\rangle \mid u_{j_{A} k_{A}^{\text {odd }} m_{A} ; j_{B} k_{B}^{\text {odd }} m_{B} ; K}^{J 00},
$$

where on the left-hand side, the composite quantum number $L \equiv$ $\left(\alpha_{0}, j_{A} k_{A}^{\text {odd }} m_{A} ; j_{B} k_{B}^{\text {odd }} m_{B} ; J K M ; P\right)$, as previously defined. To make $G_{16}$ symmetryadapted functions, we operate with $\left(E \pm P_{a b}\right)$ on Eq. (19) and obtain $A_{1}^{+}$and $B_{1}^{+}$functions. According to Eq. (17) the effect of $P_{a b}$ on a PA basis function depends on values of the 

and therefore the $G_{16} A_{1}^{+}$and $B_{1}^{+}$functions are

$$
\begin{aligned}
& \left.\left|v_{A}^{B} v_{B}^{B}, L, A_{1}^{+}\right\rangle=\frac{1}{\sqrt{2}}\left[\left|v_{A}^{B} v_{B}^{B}\right\rangle\left|u_{j_{A} k_{A}^{\text {odd }} m_{A} ; j_{B} k_{B}^{\text {odd }} m_{B} ; K}^{J 00}+(-1)^{j_{A}+k_{A}+j_{B}+k_{B}}\right| v_{B}^{B} v_{A}^{B}\right\rangle \mid u_{j_{B} \bar{k}_{B}^{\text {odd }} m_{B} ; j_{A} \bar{k}_{A}^{\text {odd }} m_{A} ; K}^{\text {on }_{B}}\right] \\
& \left.\left|v_{A}^{B} v_{B}^{B}, L, B_{1}^{+}\right\rangle=\frac{1}{\sqrt{2}}\left[\left|v_{A}^{B} v_{B}^{B}\right\rangle\left|u_{j_{A} k_{A}^{\text {odd }} m_{A} ; j_{B} k_{B}^{\text {odd }} m_{B} ; K}^{J 00}-(-1)^{j_{A}+k_{A}+j_{B}+k_{B}}\right| v_{B}^{B} v_{A}^{B}\right\rangle \mid u_{j_{B} \bar{k}_{B}^{\text {odd }} m_{B} ; j_{A} \bar{k}_{A}^{\text {odd }} m_{A} ; K}\right] .
\end{aligned}
$$

Note that if $v_{A}^{B}=v_{B}^{B}$ and $k_{A}^{\text {odd }}=k_{B}^{\text {odd }}$, one of the two functions in Eq. (20) vanishes and the surviving function's normalization constant must be changed from $1 / \sqrt{2}$ to $1 / 2$.

The example shows how to make $A_{1}^{+}$and $B_{1}^{+} G_{16}$ functions from parity-adapted functions of the form of Eq. (18). From parity-adapted basis functions with other combinations of $\left(v_{A}, v_{B}\right)$ and $\left(k_{A}, k_{B}\right)$, there are 16 types given in the the last column of Table II, one makes different $G_{16}$ symmetry-adapted functions. Note that only a single $G_{16}$ function is made from each of the last eight PA functions in the right column of Table II, because the $G_{16}$ projection operators for $E_{a}^{ \pm}$and $E_{b}^{ \pm}$do not involve $P_{a b}$. Looping through all of types of parity-adapted basis functions in the right column of Table II, one generates a complete set of $G_{16}$ functions.

Now that we have equations for the $G_{16}$ symmetry-adapted basis functions it is possible to establish the mapping that is required for packing and unpacking. To unpack a vector, we loop through components of the $G_{16}$ vector place them into the appropriate components of a PA vector. The indices of the appropriate components are pre-stored in the mapping. For the example of the previous paragraph, the appropriate components are those on the right side of Eq. (20). All other elements of the PA vector are zero. To pack a vector, one again loops through components of the $G_{16}$ vector, but obtains them from values of the appropriate components of the PA vector. The indices of the appropriate components are pre-stored in the mapping. For the example of the previous paragraph, the appropriate components are those on the right side of Eq. (20).

It is only possible to make a $G_{16}$ symmetry-adapted basis because the MVWs are isolated monomer wavefunctions. This is important because it is essential that the MVWs of monomers A and B be identical, so that they can be permuted, and essential that the MVWs have A/B symmetry. If the reference configuration for the MVWs were at the equilibrium geometry, then the $12 \mathrm{D}$ basis functions would not be invariant under all the $G_{16}$ operations, 
Publishingd one would be forced to work with a subgroup of $G_{16}$.

\section{USING THE $\boldsymbol{F}$ MATRIX METHOD TO OBVIATE STORING $\Delta V$}

The simplest way to evaluate matrix-vector products with $\left\langle v_{A}^{\prime}, v_{B}^{\prime}, L^{\prime}|\Delta V| v_{A}, v_{B}, L\right\rangle$ requires computing 12D integrals using quadrature and a grid that is a direct product of a $3 \mathrm{D}$ grid for $\left|v_{A}\right\rangle$, a $3 \mathrm{D}$ grid for $\left|v_{B}\right\rangle$, and a $6 \mathrm{D}$ grid for $|L\rangle$. Storing $\Delta V$ on a $12 \mathrm{D}$ grid is too costly. Instead, we use the $\boldsymbol{F}$ matrix idea. [53, 66-68].

The complete potential integral is

$$
\begin{aligned}
I & =\left\langle v_{A}^{\prime} v_{B}^{\prime} L^{\prime}\left|\Delta V\left(\mathbf{q}_{\mathbf{A}}, \mathbf{q}_{\mathbf{B}}, \mathbf{Q}\right)\right| v_{A}, v_{B}, L\right\rangle \\
& =\left\langle f_{\alpha_{0}^{\prime}} u_{j_{A}^{\prime} k_{A}^{\prime}{ }^{\prime} m_{A}^{\prime} ; j_{B}^{\prime} k_{B}^{\prime} m_{B}^{\prime} ; K^{\prime}}\left|\bar{V}_{v_{A}^{\prime}, v_{B}^{\prime}, v_{A}, v_{B}}(\mathbf{Q})\right| f_{\alpha_{0}} u_{j_{A} k_{A} m_{A} ; j_{B} k_{B} m_{B} ; K}\right\rangle \delta_{\alpha_{0}^{\prime}, \alpha_{0}} \delta_{J^{\prime}, J} \delta_{K^{\prime}, K},
\end{aligned}
$$

where the second line is obtained by doing the integrals over the fast monomer coordinates $\mathbf{q}_{\mathbf{A}}$ and $\mathbf{q}_{\mathbf{B}}$ and defining

$$
\bar{V}_{v_{A}^{\prime}, v_{B}^{\prime}, v_{A}, v_{B}}(\mathbf{Q})=\left\langle v_{A}^{\prime} v_{B}^{\prime}\left|\Delta V\left(\mathbf{q}_{\mathbf{A}}, \mathbf{q}_{\mathbf{B}}, \mathbf{Q}\right)\right| v_{A} v_{B}\right\rangle
$$

$\bar{V}_{v_{A}, v_{B}, v_{A}, v_{B}}(\mathbf{Q})$ is also used in Ref. 22. If $\bar{V}_{v_{A}^{\prime}, v_{B}^{\prime}, v_{A}, v_{B}}(\mathbf{Q})$ is replaced with the 6D potential obtained by starting with the $12 \mathrm{D}$ PES and setting $\mathbf{q}_{\mathbf{A}}, \mathbf{q}_{\mathbf{B}}$ to reference values, then $I$ in Eq. (21) is exactly the integral one must compute to do a rigid-monomer calculation. In appendix B, we explain in detail how to do matrix-vectors products in this case.

If one evaluates $\bar{V}_{v_{A}, v_{B}, v_{A}, v_{B}}(\mathbf{Q})$ of Eq. (22) on the $6 \mathrm{D}$ quadrature grid $Q_{\beta}$ of the slow coordinates, one obtains the $\boldsymbol{F}$ matrix,

$$
F_{v_{A}^{\prime}, v_{B}^{\prime}, v_{A}, v_{B} ; \beta}=\left\langle v_{A}^{\prime} v_{B}^{\prime}\left|\Delta V\left(\mathbf{q}_{\mathbf{A}}, \mathbf{q}_{\mathbf{B}}, Q_{\beta}\right)\right| v_{A} v_{B}\right\rangle
$$

This $\boldsymbol{F}$ matrix is similar to the $\boldsymbol{F}$ matrices used in other contracted basis calculations for $\mathrm{H}_{2} \mathrm{O}_{2}[53,69], \mathrm{CH}_{4}[67], \mathrm{CH}_{5}^{+}[68]$. All these $\boldsymbol{F}$ matrices have indices that label eigenfunctions of a Hamiltonian that depends on a subset of the coordinates (here $v_{A}^{\prime}, v_{B}^{\prime}, v_{A}, v_{B}$ ) and an index that labels a point for the remaining coordinates (here $\beta$ ). $\beta$ is a composite index that indicates values of $\alpha_{0}, \alpha_{A}-\alpha_{B}, \beta_{A}, \beta_{B}, \gamma_{A}, \gamma_{B}$. There are two important differences between the $\boldsymbol{F}$ matrices used previously and the $\boldsymbol{F}$ matrix of this paper. First, in previous papers the point index labelled a DVR basis function, and in this paper the point index labels a 
Publishi.pd $\mathrm{R}$ function for the inter-monomer coordinate and a quadrature point for the other $\mathrm{Q}$

coordinates. Second, whereas in previous papers the point index was for fast coordinates and the eigenfunction indices were for slow coordinates, in this paper the point index is for slow coordinates and the eigenfunction indices are for fast coordinates.

A matrix-vector product with $I$ in Eq. (21) can be written (for a given $\alpha_{0}$, which is omitted for brevity)

$$
\begin{gathered}
x_{v_{A}^{\prime}, v_{B}^{\prime} ; j_{A}^{\prime} k_{A}^{\prime} m_{A}^{\prime} ; j_{B}^{\prime} k_{B}^{\prime} m_{B}^{\prime} ; K}^{\prime}=\sum_{v_{A}, v_{B}} \sum_{p_{1}, p_{2}} T_{j_{A}^{\prime}, p_{1}}^{m_{A}^{\prime}, k_{A}^{\prime}} T_{j_{B}^{\prime}, p_{2}}^{m_{B}^{\prime}, k_{B}^{\prime}} \sum_{k_{A} m_{A} ; k_{B} m_{B} ; K} \\
\times I_{m_{A}^{\prime} m_{B}^{\prime} k_{A}^{\prime} k_{B}^{\prime}, m_{A}, m_{B} k_{A} k_{B}}^{v_{j_{A}}^{\prime}, v_{A}, v_{B} ; p_{1}, p_{2}} \sum_{j_{A} j_{B}} T_{j_{A}, p_{1}}^{m_{A}, k_{A}} T_{j_{B}, p_{2}}^{m_{B}, k_{B}} x_{v_{A}, v_{B} ; j_{A} k_{A} m_{A} ; j_{B} k_{B} m_{B} ; K},
\end{gathered}
$$

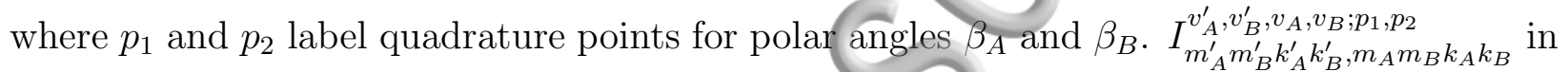
the middle is a 9D integral obtained from Eq. (21) by omitting the $d_{m_{A} k_{A}}^{j_{A}}$ (see Appendix A) functions and integrating over $q_{A}, q_{B}$, and three dihedral angles. The three dihedral angles are: $\tilde{\alpha}=\alpha_{A}-\alpha_{B}, \gamma_{A}$ and $\gamma_{B}$. Evaluation of the integral is discussed in detail in Appendix B. In the appendix, the $v_{A}^{\prime}, v_{B}^{\prime}, v_{A}, v_{B}$ superscripts are not there because the same integral is discussed for a rigid-monomer calculation. There are three ways of determining how $E^{*}$ affects $\alpha_{A / B}, \beta_{A / B}$, and $\gamma_{A / B}$. In this paper we use case II of Ref. 55. It is therefore possible to use equations similar to those in Refs. $70-72$ for methane to compute $I_{m_{A}^{\prime} m_{B}^{\prime} v_{A}^{\prime}, v_{A}^{\prime}, v_{B}, v_{B} ; p_{1}, p_{2}}^{v_{A} m_{B} k_{A} k_{B}}$. In methane there are two dihedral-like angles and in water dimer there are three. After evaluating the sums over $j_{A}$ and $j_{B}$ and denoting the result $u_{m_{A} k_{A} k_{B}}$ (other indices are omitted, $m_{B}$ is replaced with $K-m_{A}$, and the potential integral is diagonal in $K$ ) we use FFTs to calculate

$$
u_{m_{A}^{\prime} k_{A}^{\prime} k_{B}^{\prime}}^{\prime}=\sum_{v_{A}, v_{B}} \operatorname{iFFT}\left(q_{1}, q_{2}, q_{3} ; m_{A}^{\prime} k_{A}^{\prime} k_{B}^{\prime}\right) F_{v_{A}^{\prime}, v_{B}^{\prime}, v_{A}, v_{B} ; \beta} \mathrm{FFT}\left(m_{A} k_{A} k_{B} ; q_{1}, q_{2}, q_{3}\right) u_{m_{A} k_{A} k_{B}}
$$

where $q_{1}, q_{2}, q_{3}$ label quadrature points for $\tilde{\alpha}, \gamma_{A}$, and $\gamma_{B}$ and $\beta$ represents $p_{1}, p_{2}, q_{1}, q_{2}, q_{3}, \alpha_{0}$. FFT and iFFT are a FFT and an inverse FFT. This equation is closely related to Eq. 24 of Ref. 72, but the FFT is now 3D rather than 2D. The FFTs are done differently depending on whether $K=0$ or $K \neq 0$ and depending on the value of $(-1)^{J+P}$. The different cases can be obtained from equations B11, B13, B15 for $I$ in appendix B by replacing $V_{q_{1}, q_{2}, q_{3}}$ which is the potential evaluated at a $\tilde{\alpha}, \gamma_{A}$, and $\gamma_{B}$ quadrature point, with $F_{v_{A}^{\prime}, v_{B}^{\prime}, v_{A}, v_{B} ; \beta}$. From $u_{m_{A}^{\prime} k_{A}^{\prime} k_{B}^{\prime}}^{\prime}$ we compute $x_{v_{A}^{\prime}, v_{B}^{\prime} ; j_{A}^{\prime} k_{A}^{\prime} m_{A}^{\prime} ; j_{B}^{\prime} k_{B}^{\prime} m_{B}^{\prime} ; K}$ by summing over $p_{1}$ and $p_{2}$ and $v_{A}$ and $v_{B}$. To 
Publishisignplify the equations in the rest of the paper, we combine $T_{j_{w}, p_{w}}^{m_{w}, k_{w}}, w=A, B$ and the $3 \mathrm{D}$ FFT in Eq. (24) and Eq. (25) into a single transformation matrix which is

$$
\mathcal{T}_{j_{A} k_{A} m_{A}, j_{B} k_{B} m_{B}, \beta}=\mathrm{FFT}\left(m_{A} k_{A} k_{B} ; q_{1}, q_{2}, q_{3}\right) T_{j_{A}, p_{1}}^{m_{A}, k_{A}} T_{j_{B}, p_{2}}^{m_{B}, k_{B}}
$$

Its inverse is

$$
\mathcal{T}_{\beta, j_{A} k_{A} m_{A}, j_{B} k_{B} m_{B}}^{-1}=T_{j_{A}, p_{1}}^{m_{A}, k_{A}} T_{j_{B}, p_{2}}^{m_{B}, k_{B}} \cdot \operatorname{iFFT}\left(q_{1}, q_{2}, q_{3} ; m_{A} k_{A} k_{B}\right)
$$

$\mathcal{T}$ transforms a vector labelled by basis indices $\left(j_{A} k_{A} m_{A}, j_{B} k_{B} m_{B} ; K\right)$ to a vector labelled by a point, $\beta . \mathcal{T}^{-1}$ does the reverse transform.

We do not actually compute $I$ in Eq. (21). To use either the Lanczos or the Arnoldi algorithm, it is only necessary to evaluate matrix-vector products. Ro-vibrational spectra have been computed with iterative algorithms for many years. [26, 73-78] The $\Delta V$ matrixvector product in the contracted basis is computed in three steps,

$$
\begin{aligned}
y_{v_{A}, v_{B}, \beta} & =\sum_{j_{A} k_{A} m_{A} ; j_{B} k_{B} m_{B} ; K} \mathcal{T}_{j_{A} k_{A} m_{A}, j_{B} k_{B} m_{B}, \beta} x_{v_{A}, v_{B}, j_{A} k_{A} m_{A}, j_{B} k_{B} m_{B} ; K} \\
y_{v_{A}^{\prime}, v_{B}^{\prime}, \beta}^{\prime} & =\sum_{v_{A}, v_{B}} F_{v_{A}^{\prime}, v_{B}^{\prime}, v_{A}, v_{B} ; \beta} y_{v_{A}, v_{B}, \beta} \\
x_{v_{A}^{\prime}, v_{B}^{\prime}, j_{A}^{\prime} k_{A}^{\prime} m_{A}^{\prime}, j_{B}^{\prime} k_{B}^{\prime} m_{B}^{\prime} ; K}^{\prime} & =\sum_{\beta} \mathcal{T}_{\beta, j_{A}^{\prime} k_{A}^{\prime} m_{A}^{\prime}, j_{B}^{\prime} k_{B}^{\prime} m_{B}^{\prime}} y_{v_{A}^{\prime}, v_{B}^{\prime}, \beta} .
\end{aligned}
$$

The first and third steps are the same as in a 6D rigid-monomer calculation. The second step would be same if $F_{v_{A}^{\prime}, v_{B}^{\prime}, v_{A}, v_{B} ; \beta}$ were replaced by the potential evaluated at a quadrature point, $V_{\beta}$, and the sums over $v_{A}$ and $v_{B}$ were removed.

\section{COMPUTING THE $F$ MATRIX}

We use a pruned 3D DVR basis, $\xi_{X}$, to compute $\left|v_{X}\right\rangle$, eigenstates of the 3D monomer vibrational Hamiltonian $H^{X}$ defined in Eq. (7). We retain DVR functions peaked at points at which the PES is less than a threshold value. Elements of the matrix of eigenvectors of the DVR representation of $H^{X}$ are $\left\langle\xi_{X} \mid v_{X}\right\rangle . \xi_{X}$ is a collective label for a 3D DVR function in the pruned basis. To compute $\boldsymbol{F}$ we insert a complete set of DVR states into the middle of Eq. (23)

$$
F_{v_{A}^{\prime}, v_{B}^{\prime}, v_{A}, v_{B} ; \beta}=\sum_{\xi_{A}, \xi_{B}}\left\langle\xi_{A} \mid v_{A}^{\prime}\right\rangle^{*}\left\langle\xi_{B} \mid v_{B}^{\prime}\right\rangle^{*} \Delta V\left(q_{\xi_{A}}, q_{\xi_{B}} ; Q_{\beta}\right)\left\langle\xi_{A} \mid v_{A}\right\rangle\left\langle\xi_{B} \mid v_{B}\right\rangle .
$$


Publishi.figaluating this sum as it is written requires $N_{v_{A}}^{2} N_{v_{B}}^{2} N_{\xi_{A}} N_{\xi_{B}} N_{\beta}$ multiplications. It is more efficient to do it in two steps:

$$
X_{v_{A}^{\prime}, v_{A}, \xi_{B}, \beta}=\sum_{\xi_{A}}\left\langle\xi_{A} \mid v_{A}^{\prime}\right\rangle^{*} \Delta V\left(q_{\xi_{A}}, q_{\xi_{B}} ; Q_{\beta}\right)\left\langle\xi_{A} \mid v_{A}\right\rangle
$$

and

$$
F_{v_{A}^{\prime}, v_{B}^{\prime}, v_{A}, v_{B} ; \beta}=\sum_{\xi_{B}}\left\langle\xi_{B} \mid v_{B}^{\prime}\right\rangle^{*}\left\langle\xi_{B} \mid v_{B}\right\rangle X_{v_{A}^{\prime}, v_{A}, \xi_{B}, \beta} .
$$

The cost of steps one and two is $N_{v_{A}}^{2} N_{\xi_{A}} N_{\xi_{B}} N_{\beta}$ and $N_{v_{A}}^{2} N_{v_{B}}^{2} N_{\xi_{B}} N_{\beta}$, respectively. Compared to the cost of the direct computation, they are cheaper by factors of $N_{v_{B}}^{2}$ and $N_{\xi_{A}}$. Because we expect $N_{v_{B}}^{2}<N_{\xi_{A}}$, the cost of the second step should be smaller than that of the first step.

The memory cost of the entire calculation is dominated by the need to store the $\boldsymbol{F}$ matrix. It is therefore important to reduce its size, without degrading the quality of calculated levels. We reduce its size by constraining indices $v_{A}$ and $v_{B}$ so that $\mathcal{E}_{v_{A}}^{A}+\mathcal{E}_{v_{B}}^{B}$ is less than a cut-off value, $E_{\text {cut }}=3800 \mathrm{~cm}^{-1}$. We start with the direct product $\left|v_{A}, v_{B}\right\rangle$ basis made from the five monomer functions $\left|v_{X}\right\rangle$ for which $\mathcal{E}_{v_{X}}^{X} \leq E_{\text {cut }}$. After removing the product functions whose energy is greater than $E_{\text {cut }}=3800 \mathrm{~cm}^{-1}$, the resulting pruned basis has only 10 functions, compared with 25 functions if the basis is not pruned. Using the pruned basis therefore reduces the size of the $\boldsymbol{F}$ matrix by a factor of 6.25 .

\section{METHODS TO MAKE THE CALCULATION MORE EFFICIENT}

In this section, we discuss three methods to make the calculation more efficient. They reduce the cost of computing and storing the $\boldsymbol{F}$ matrix which is the most expensive part of the calculation. The methods in the first and third sub-sections reduce the number of $\mathbf{Q}$ points and hence the size of the $\boldsymbol{F}$ matrix. In the second sub-section, we present ideas that make it possible to obtain all $\boldsymbol{F}$ matrix elements from a symmetry-unique set of $\boldsymbol{F}$ matrix elements. 


\section{PublishingA. Optimization of the 6D basis functions for the rigid monomer calculation}

The number of $\mathbf{Q}$ points $(\beta)$ is a product of the number of DVR functions for $r_{0}$ and the number of quadrature points required for the $\alpha_{A}-\alpha_{B}, \beta_{A}, \beta_{B}, \gamma_{A}, \gamma_{B}$ coordinates. We use Gauss Legendre points for polar angles $\beta_{A}$ and $\beta_{B}$ and Fourier points for dihedral angles with the first point at zero. The required number of Gauss Legendre point $N_{\theta}$ and Fourier points $N_{\phi}$ is determined by the maximum values of $\left(j_{A}, j_{B}\right),\left(m_{A}, m_{B}\right),\left(k_{A}, k_{B}\right)$ which are $j_{\max }, m_{x}$ and $k_{x}$, respectively. Assuming $j_{\max }=m_{x}=k_{x}$, we choose $N_{\theta} \geq j_{\max }+1$ and $N_{\phi} \geq 2 j_{\max }+1$. $N_{\phi}$ may also be chosen to increase the the efficiency of the FFT[71]. See Table III. We can reduce the number of $\mathbf{Q}$ points by optimising the DVR for $r_{0}$. It is common to use a potential-optimized DVR. [79, 80] In this paper we have, instead, used a tri-diagonal Morse(TDM) DVR basis.[79] It is smaller than the PODVR basis of equivalent accuracy. The TDM basis and the selection of its parameters is discussed in section VIIIA.

\section{B. Use symmetry to compute only the unique $\boldsymbol{F}$ matrix elements}

There are simple relations among $\boldsymbol{F}$ matrix elements that make it possible to avoid computing and storing all of them. They are due to symmetry. When a symmetry operator, $\hat{O}$, is applied to $F_{v_{A}^{\prime}, v_{B}^{\prime}, v_{A}, v_{B} ; \beta}$ it is unchanged. In general, a symmetry operator will affect both $\mathbf{q}$ and $\mathbf{Q}$ coordinates. We shall indicate coordinate values after application of a symmetry operator with a superscript. For example, the (12) operation changes the coordinates $\left(\mathbf{q}_{\mathbf{A}}, \mathbf{q}_{\mathbf{B}} ; Q_{\beta}\right)$ to $\left(\mathbf{q}_{\mathbf{A}}^{(\mathbf{1 2})}, \mathbf{q}_{\mathbf{B}} ; Q_{\beta^{(12)}}\right)$. Of course,

$$
(12) \Delta V\left(\mathbf{q}_{\mathbf{A}}, \mathbf{q}_{\mathbf{B}} ; Q_{\beta}\right)=\Delta V\left(\mathbf{q}_{\mathbf{A}}^{(12)}, \mathbf{q}_{\mathbf{B}} ; Q_{\beta^{(12)}}\right) .
$$

From Eq. (13) and Eq. (32), one finds that $\boldsymbol{F}$ matrix elements for the two slow points $Q_{\beta}$ and $Q_{\beta(12)}$ are linked,

$$
F_{v_{A}^{\prime}, v_{B}^{\prime}, v_{A}, v_{B} ; \beta}=s\left(v_{A}^{\prime}\right) s\left(v_{A}\right) F_{v_{A}^{\prime}, v_{B}^{\prime}, v_{A}, v_{B} ; \beta^{(12)}} .
$$


Publishíngis means that if we compute and store the LHS we can obtain $F_{v_{A}^{\prime}, v_{B}^{\prime}, v_{A}, v_{B} ; \beta^{(12)}}$ for free. In the same manner, we find,

$$
\begin{aligned}
& F_{v_{A}^{\prime}, v_{B}^{\prime}, v_{A}, v_{B} ; \beta}=s\left(v_{B}^{\prime}\right) s\left(v_{B}\right) F_{v_{A}^{\prime}, v_{B}^{\prime}, v_{A}, v_{B} ; \beta(34)} \\
& F_{v_{A}^{\prime}, v_{B}^{\prime}, v_{A}, v_{B} ; \beta}=F_{v_{B}^{\prime}, v_{A}^{\prime}, v_{B}, v_{A} ; \beta^{P} a b} \\
& F_{v_{A}^{\prime}, v_{B}^{\prime}, v_{A}, v_{B} ; \beta}=F_{v_{A}^{\prime}, v_{B}^{\prime}, v_{A}, v_{B} ; \beta^{E^{*}}} .
\end{aligned}
$$

In this paper, we use the parity-adapted basis and therefore only have points with $\gamma_{B}$ to $[0, \pi]$. This reduces the size of the $\beta$ grid by a factor of two. In addition, by using equations like Eq. (33) and Eq. (34), it is only necessary to compute one quarter of the $F_{v_{A}^{\prime}, v_{B}^{\prime}, v_{A}, v_{B} ; \beta}$. $F_{v_{A}^{\prime}, v_{B}^{\prime}, v_{A}, v_{B} ; \beta}$ in three of the four quadrants in the $\gamma_{A} \tilde{\alpha}$ plane are obtained, for free, from those in the remaining quadrant by using the three operators (12), (34)*, and (12)(34)*. Equations like those of Eq. (34), but for $(12),(34)^{*}$, and $(12)(34)^{*}$, can easily be obtained from them. We could also utilize $P_{a b}$ symmetry to reduce the the number of $\boldsymbol{F}$ matrix elements by another factor of two but choose not to, for simplicity. The reason we choose (12), (34)*, and (12)(34)* is that the corresponding points, $\beta^{(12)}, \beta^{(34)^{*}}$, and $\beta^{(12)(34)^{*}}$ are all on the slow grid used in the calculation. For some operators $\beta^{\hat{O}}$ is not on the grid because we use the parity-adapted basis. From $(12),(34)^{*}$, and $(12)(34)^{*}$ it is possible to obtain $F_{v_{A}^{\prime}, v_{B}^{\prime}, v_{A}, v_{B} ; \beta}$ for which $\beta$ corresponds to a point with $\left(\gamma_{B}, \gamma_{A}, \alpha_{A}-\alpha_{B}\right)$ in $([0, \pi],[0,2 \pi],[0,2 \pi])$ from $F_{v_{A}^{\prime}, v_{B}^{\prime}, v_{A}, v_{B} ; \beta}$ for which $\beta$ corresponds to a point with $\left(\gamma_{B}, \gamma_{A}, \alpha_{A}-\alpha_{B}\right)$ in $([0, \pi],[0, \pi],[0, \pi])$. Table IV shows how coordinates are affected by symmetry operations. Using it, one can verify that (12), (34)*, and $(12)(34)^{*}$ keep $\gamma_{B}$ in $[0, \pi]$. Similar ideas are also used to decrease the number of stored potential values for the $6 \mathrm{D}$ calculation, but the memory reduction is much less important. It was mentioned in Ref. 25 that it is possible to store only non-symmetry-equivalent grid points in a rigid monomer computation, but no details were given. For $\mathrm{H}_{2} \mathrm{O}-\mathrm{D}_{2} \mathrm{O}$, we would use only the symmetry operation (12). The range of $\left(\gamma_{B}, \gamma_{A}, \alpha_{A}-\alpha_{B}\right)$ for which $\boldsymbol{F}$ matrix elements are computed would be $([0, \pi],[0, \pi],[0,2 \pi])$. $\boldsymbol{F}$ matrix elements in the full range of $\chi_{A}$ could be recovered by using (12). 
In the $6 \mathrm{D}$ calculation, we use the common idea of replacing the potential, where it is larger than a threshold value, by a ceiling value. [81] [18, 53] We denote this modified potential $\tilde{V}^{6 D}(\mathbf{Q})$. It is assumed that wavefunctions on the original potential $V^{6 D}(\mathbf{Q})$ and on the modified potential $\tilde{V}^{6 D}(\mathbf{Q})$ are almost identical. When evaluating integrals by quadrature, all points for which $\tilde{V}^{6 D}(\mathbf{Q})$ returns the ceiling value can be omitted One way to understand the ceiling idea is to replace $\left\langle L^{\prime}\left|\tilde{V}^{6 D}(\mathbf{Q})\right| L\right\rangle$ with $\left\langle L^{\prime}\left|\tilde{V}^{6 D}(\mathbf{Q})-V_{\text {ceil }}\right| L\right\rangle+V_{\text {ceil }}$ and then it is clear that the integrand is zero at ceiling points.

An idea similar to the ceiling idea of the previous paragraph can be used to obviate calculating and storing $F_{v_{A}^{\prime}, v_{B}^{\prime}, v_{A}, v_{B} ; \beta}$ when $V_{r e f}^{6 \mathrm{D}}(\mathbf{Q})=\mathbf{V}\left(\mathbf{q}_{\mathbf{A}}^{\text {ref }}, \mathbf{q}_{\mathbf{B}}^{\text {ref }}, \mathbf{Q}_{\beta}\right)$ is larger than a ceiling value. It saves a significant amount of computer time and memory. $V_{\text {ref }}^{6 \mathrm{D}}$ retains the full $G_{16}$ symmetry. The $12 \mathrm{D}$ potential integral as given in Eq. (21) is

$$
I=\left\langle v^{\prime}, L^{\prime}\left|\Delta V\left(\mathbf{q}_{\mathbf{A}}, \mathbf{q}_{\mathbf{B}}, \mathbf{Q}\right)\right| v, L\right\rangle=\sum_{\beta}\left\langle L^{\prime} \mid \beta\right\rangle F_{v^{\prime}, v, \beta}\langle\beta \mid L\rangle .
$$

To reject points, we define $V_{r e f}^{6 D}(\mathbf{Q})=V\left(\mathbf{q}_{\mathbf{A}}^{\text {ref }}, \mathbf{q}_{\mathbf{B}}^{\text {ref }}, \mathbf{Q}\right)$, where $q_{X}^{\text {ref }}(X=A, B)$ is the equilibrium value of $\mathbf{q x}_{\mathbf{x}}$ for an isolated monomer. One could use $V_{r e f}^{6 D}(\mathbf{Q})$ with a $\mathrm{KEO}$ that is a sum of $T_{i n t}+T_{r}^{A}+T_{r}^{B}$ to compute a set of inter-monomer wavefunctions. Modifying $V_{r e f}^{6 D}(\mathbf{Q})$, by introducing a ceiling, as explained in the previous paragraph, one can argue that quadrature points on the ceiling are not necessary. We assume points that are not necessary for this inter-monomer calculation are also not necessary for the $12 \mathrm{D}$ calculation. We divide the full point set into two groups: points for which $V_{r e f}^{6 D}(\mathbf{Q})$ is less than the ceiling $\left(\beta^{\prime}\right)$ and points for which $V_{r e f}^{6 D}(\mathbf{Q})$ is greater than the ceiling $\left(\beta^{\prime \prime}\right)$,

$$
I=\sum_{\beta^{\prime}}\left\langle L^{\prime} \mid \beta^{\prime}\right\rangle F_{v^{\prime}, v, \beta^{\prime}}\left\langle\beta^{\prime} \mid L\right\rangle+\sum_{\beta^{\prime \prime}}\left\langle L^{\prime} \mid \beta^{\prime \prime}\right\rangle F_{v^{\prime}, v, \beta^{\prime \prime}}\left\langle\beta^{\prime \prime} \mid L\right\rangle .
$$

If $\beta^{\prime \prime}$ points can be omitted, then in second term $\Delta V$ can be replaced with the constant $V_{\text {ceil }}$ and one obtains

$$
I=\sum_{\beta^{\prime}}\left\langle L^{\prime} \mid \beta^{\prime}\right\rangle F_{v^{\prime}, v, \beta^{\prime}}\left\langle\beta^{\prime} \mid L\right\rangle+\sum_{\beta^{\prime \prime}}\left\langle L^{\prime} \mid \beta^{\prime \prime}\right\rangle V_{\text {ceil }} \delta_{v^{\prime}, v}\left\langle\beta^{\prime \prime} \mid L\right\rangle .
$$

Now add and subtract $\sum_{\beta^{\prime}} V_{\text {ceil }} \delta_{v^{\prime}, v}$ to obtain

$$
I=\sum_{\beta^{\prime}}\left\langle L^{\prime} \mid \beta^{\prime}\right\rangle\left(F_{v^{\prime}, v, \beta^{\prime}}-V_{\text {ceil }} \delta_{v^{\prime}, v}\right)\left\langle\beta^{\prime} \mid L\right\rangle+V_{\text {ceil }} \delta_{v^{\prime}, v} \delta_{L^{\prime}, L}
$$


Publishifagalogous to the use of ceiling for potential integral, the diagonal $\boldsymbol{F}$ matrix elements are shifted and only $\boldsymbol{F}$ matrix elements for $Q_{\beta}$ points for which $V_{r e f}^{6 D}(\mathbf{Q})$ is less than the ceiling value are needed in the summation (over $\beta^{\prime}$ ). This reduces the number of $\boldsymbol{F}$ matrix elements which must be computed.

An $\boldsymbol{F}$ matrix was used first to compute vibrational levels of $\mathrm{CH}_{4}[67]$ and $\mathrm{CH}_{5}^{+}[68]$ in a $|b s\rangle$ basis, where $|b\rangle$ is a bend contracted basis and $|s\rangle$ is a stretch contracted basis. In that case,

$$
\left\langle b^{\prime} s^{\prime}|\Delta V(\theta, r)| b s\right\rangle=\sum_{\alpha}\left\langle s^{\prime} \mid \alpha\right\rangle F_{b^{\prime}, b, \alpha}\langle\alpha \mid s\rangle
$$

In those papers, $\alpha$ labels a function in the DVR basis used to compute $|s\rangle$ and the number of $\boldsymbol{F}$ matrix elements that must be computed and stored is reduced by discarding DVR functions and therefore $\boldsymbol{F}$ matrix elements. The discarded DVR functions are not required for computing $|s\rangle$. In this paper we do not discard; instead we use the ceiling idea.

\section{CALCULATION DETAILS}

\section{A. Potential}

The CCpol-8sf[26] PES used in this study was downloaded form the supporting information of Ref. 31. Ref. 31 discussed several PESs (see its Table 3) and renamed CCpol-8sf as CCpol-8sfIR[2012]. There are different versions of the CCpol-8sf PES for different choices of the monomer-fixed frame. One version is used with Radau coordinates for the monomers and the so-called $f=1$ embedding (a bisector embedding based on Radau vectors). Another version is used with the Eckart frame. We choose the former embedding as does Ref. 26. The coordinates and energy for the equilibrium structure are given in Fig. 1.

\section{B. $6 \mathrm{D}$ rigid-monomer basis optimization and 6D energy levels}

As discussed in section VII A, an optimised 6D basis for the rigid monomer calculation has the important benefit that it is necessary to compute $\boldsymbol{F}$ matrix elements for fewer $\mathbf{Q}_{\beta}$ points. We optimize the $6 \mathrm{D}$ basis by choosing the best possible DVR basis for $r_{0}$, which is discussed in this subsection. Using the most accurate CCpol-8s 6D PES[21], we first did 
Publishibgrgchmark calculations with a large number of sine DVR points (120)[82-84] between 4.2 and 10.0 Bohr and a large bend basis defined by $j_{x}=12, N_{\theta}=16$ and $N_{\phi}=32 . N_{\theta}$ and $N_{\phi}$ are numbers of quadrature points for the $\theta$-type and $\phi$-type angles. This benchmark basis has 24 million functions for $J=0$ even parity. Against this benchmark basis results, we tested PODVR bases using a cut reference potential and tri-diagonal Morse (TDM) DVR bases. A PODVR is often a good choice, however, for the $6 \mathrm{D}$ water dimer calculations a TDM basis is better. The three TDM parameters, $D_{e}, \omega$, and $r_{e}$, are varied to find the best $6 \mathrm{D} E^{+}$levels. The final TDM DVR basis parameters-are: $D_{e}=1785 \mathrm{~cm}^{-1}, \omega=145$ $\mathrm{cm}^{-1}$, and $r_{e}=5.504$ Bohr. A fourth TDM DVR basis parameter $\alpha$ depends on the other parameters ( $\alpha$ appears in the associated Laguerre polynomial $L_{n}^{\alpha}$ ). As discussed in Ref. 47 , we follow Wei and Carrington's choice[79] and set, $\alpha=A-2 N_{r_{0}}$, with $A=4 D_{e} / \omega_{e}=44.35$. This ensures that the first $N_{r_{0}}$ bound states of the Morse Hamiltonian (the number of bound states of the Morse Hamiltonian is $[A / 2]=22$, which is much larger than $N_{r_{0}}$ ) are exactly reproduced by the TDM basis. Compared to the $E^{+} J=0$ benchmark levels, a TDM DVR basis with $N_{r_{0}}=7$ functions performs as well as a PODVR basis with $N_{r_{0}}=9$ functions and the errors are smaller than $0.01 \mathrm{~cm}^{-1}$ up to $150 \mathrm{~cm}^{-1}$, compared to the benchmark levels. See Table V. Thus, the TDM $\boldsymbol{F}$ matrix is $22 \%$ smaller than its PODVR counterpart.

The $6 \mathrm{D}$ calculation is not the focus of this paper, however, in the Supplementary Material (SM), Tables V, VI, and VII we present the $J=0,1$, and $26 \mathrm{D}$ levels computed with a basis defined by $j_{x}=14, N_{\theta}=15$ and $N_{\phi}=30$ and $N_{r_{0}}=9$ PODVR points. These levels are converged to within $0.001 \mathrm{~cm}^{-1}$ for levels up to $150 \mathrm{~cm}^{-1}$. Because the bend basis is larger, the $6 \mathrm{D}$ levels in the SM are even more accurate than than those of Table V. The accuracy is confirmed by comparing with levels computed with the same bend basis but 120 sine DVR basis. This benchmark basis has 49 million functions for $J=0$, even parity.

There are two previous calculations on CCpol-8s 6D PES[21, 26]. Although neither paper reports levels directly, we believe our levels are better converged. A comparison of $6 \mathrm{D} K=0$ and $K=1$ fork origins and interchange tunneling splittings, both of which are derived from levels, is shown in SM Table VIII. Our ZPE - $1094.23 \mathrm{~cm}^{-1}$ is smaller than $-1093.54 \mathrm{~cm}^{-1}$ computed in Ref. 21 and $-1094 \mathrm{~cm}^{-1}$ computed (6D) in Ref. 26.

In the $6 \mathrm{D}$ calculation, the masses are $m_{\mathrm{H}}=1.007825035$ and $m_{\mathrm{O}}=15.99491463$ in atomic mass units. The rotational constants for rigid $\mathrm{H}_{2} \mathrm{O}$ are $A=27.8806, B=14.5216$ and $C=$ 
Publishipg.g $78 \mathrm{~cm}^{-1}$, taken from Ref. 19. The same masses are used in the 12D calculations.

\section{12D convergence tests and cost of calculations}

We have tested the convergence of the levels with respect to the three most important convergence parameters: (i) the ceiling value; (ii) the size of the $6 \mathrm{D}$ inter-monomer basis; (iii) $N_{v}$, the size of the MVW basis. The size of the basis used to compute the MVW is large and is not varied. The bases we used are defined in Table III. Bases 1 and 2 have the same number of intra-monomer basis functions. Basis 1 has more basis inter-monomer functions (functions of $\mathbf{Q}$ ) than basis 2. $E^{+}$levels are selected to do all the convergence tests. Levels computed with different bases are given in Table VI.

The ceiling value (i) determines the number of quadrature points for which $F_{v^{\prime}, v, \beta}$ is computed. To test convergence with respect to the ceiling value, we compare levels computed with $V_{\text {ceil }}=1000 \mathrm{~cm}^{-1}$ (Basis 1) and with $V_{\text {ceil }}=30000 \mathrm{~cm}^{-1}$ (Basis 1f). Whereas Basis 1f uses all the $\mathbf{Q}$ quadrature points, Basis 1 keeps $83 \%$ of the $\mathbf{Q}$ points. Basis 1 levels up to $200 \mathrm{~cm}^{-1}$ are accurate to within $0.01 \mathrm{~cm}^{-1}$. We therefore know that it is safe to use the smaller $\mathbf{Q}$ point set and smaller $\boldsymbol{F}$ matrix. To test the size of the $6 \mathrm{D}$ basis (ii), we compare levels computed with Basis 1 and Basis 2, which have different numbers of $\mathbf{Q}$ functions. In all the calculations $k_{x}=m_{x}=j_{x}$. For levels up to $200 \mathrm{~cm}^{-1}$, convergence errors are less than $0.3 \mathrm{~cm}^{-1}$. To test the influence of $N_{v}$ (iii), we use Basis 1 and increase $N_{v}$ from 10 to 48. The levels differ by/less than $0.06 \mathrm{~cm}^{-1}$ up to $200 \mathrm{~cm}^{-1}$. Basis 1 with $N_{v}=10$ gives a good balance between accuracy and basis size. We use it to calculate $J>0$ levels. We use it to calculate all inter-molecular and intra-molecular levels presented in this paper. The size of Basis 1, with $N_{v}=10$, for $J=0$, and even parity, is 13.9 million. Using the SAL algorithm, one can simultaneously obtain energy levels for all 5 irreps up to $300 \mathrm{~cm}^{-1}$ with 1000 iterations. Each iteration takes 6.4 seconds on 8 cores. The potential matrix-vector products are parallelized over $v_{A}, v_{B}, \alpha_{0}$, and $K$. Computing low-lying levels with the SAL is therefore relatively quick.

The most time consuming part of the calculation is computing the $\boldsymbol{F}$ matrix. This cost is mitigated by using MPI to parallelize by distributing millions of $\beta$ values over thousands of cores. An MPI process first reads the full set of MVW from disk. It then receives a set of 
Publishipg $\Delta V\left(\mathbf{q}_{\mathbf{A}}, \mathbf{q}_{\mathbf{B}}, \mathbf{Q}^{\beta}\right)$ vectors, one for each of its $\beta$ values, and computes the corresponding portion of the $\boldsymbol{F}$ matrix. Each MPI process is independent. The parallelization efficiency is nearly $100 \%$. Using 1024 cores, it takes about 1.5 days to compute the $\boldsymbol{F}$ matrix for Basis 1 with $N_{v}=10$. Storing the $\boldsymbol{F}$ matrix (after a factor of 4 reduction achieved utilizing the (12), (34)* and (12)(34)* symmetry operations) requires only 617 MB memory.

Using ARPACK allows us to get higher levels and wavefunctions more quickly than would be possible with the SAL, but at the cost of requiring more memory. We store 4500 Arnoldi vectors in memory. The memory cost is mitigated by using the symmetry-adapted basis. For example, with Basis 1 and $N_{v}=10$, to get the lowest $3000 J=0 A_{1}^{+}$levels, the size of an Arnoldi vector for a single $G_{16}$ irrep is 1.75 million, about 8 times smaller than that of parity-adapted vector. The memory cost is 67 GB and the-calculation takes 4 days and 12 hours on 48 cores.

\section{12D RESULTS}

We present our calculated levels in two subsections. The inter-molecular levels are in the first subsection and water bend intra-molecular levels are in the second subsection. Calculating more intra-molecular levels is costly due to the high density of states.

\section{A. Inter-molecular vibrational-rotational-tunneling (VRT) levels}

The degeneracy pattern of the ro-vibrational levels of water dimer is well-known. [13, 85] Owing to the fact that there are 8 equivalent wells, each inter-molecular state is split into 8 states. Two of the 8 states are doubly degenerate and each inter-molecular level therefore splits into 6 levels. They occur as two triple forks, one is for states with even parity and the other is for states with odd parity. In each triple fork, the middle prong is an $E$ state and the top and bottom prongs are $A$ or $B$. The difference between the averages of the $A / B$ pairs of the two triple forks is known as the acceptor tunnelling splitting. The difference between

the $A$ and $B$ levels within a triple fork is known as an interchange tunnelling splitting. The difference between the average of the $B$ and $A$ levels of a triple fork and the $E$ level of the same fork is called a bifurcation tunnelling splitting. This energy level pattern is discussed in detail in Refs. 21, 26. 
Publishing There are several ways one can compare different theoretical results. The most direct way is to compare levels. This gives an explicit and detailed estimate of convergence error and the effect of approximations. The $12 \mathrm{D}$ calculation is costly and it therefore makes sense to report the actual levels. One can also compare tunnelling splittings and other energy level differences. They also make it possible to compare theory with experiment. Because of the large number of levels, in almost of all previous theoretical papers, origins for each of the triple forks, inferred from the computed levels, are reported. From the origins one can calculate an acceptor tunnelling splitting. See Refs. 21 and 26. These origins and tunneling splittings can also be compared with their experimental counterparts. Using the origins has the advantage that it is concise and allows one to quickly assess the general quality of the calculations. In this subsection, we first compare the actual levels we compute with those obtained with the $[6+6] \mathrm{D}$ method. [26] We then compare origins and tunneling splittings with those of the $[6+6] \mathrm{D}$ method and with experiment.

We first compare levels. A complete set of $J=0,1$ and 2 levels is presented in SM Tables I, II and III. This will facilitate comparison with future calculations. The [6+6]D levels were not published, but were kindly provided to us by Leforestier[86]. Our best ZPE, relative to the bottom of the well, obtained with basis 1f, is $8159.758 \mathrm{~cm}^{-1}$ (Table VI). From this $\mathrm{ZPE}$ and the $\mathrm{ZPE}$ of an isolated water monomer one finds $D_{0}=2 E_{\mathrm{ZPE}, \mathrm{H}_{2} \mathrm{O}}-E_{\mathrm{ZPE}}=$ $1109.744 \mathrm{~cm}^{-1}$, where we have used $E_{\mathrm{ZPE}, \mathrm{H}_{2} \mathrm{O}}=4634.751 \mathrm{~cm}^{-1}$, which has been computed on the CCpol-8sf PES. This $D_{0}$ is larger than $D_{0}=1108.265 \mathrm{~cm}^{-1}$ obtained with the [6+6]D method[26] on the same PES, and both are in good agreement with the experimental $D_{0}=$ $1105 \pm 10 \mathrm{~cm}^{-1}$ recently measured by Rocher-Casterline et al.[10]. Overall, our 12D levels are in good agreement with the $[6+6] \mathrm{D}$ levels. Our ZPE is lower by $1.48 \mathrm{~cm}^{-1}$ as indicated by $D_{0}$ given above. The differences of levels relative to the ZPE between the two calculations are within $0.5 \mathrm{~cm}^{-1}$, for levels up to $200 \mathrm{~cm}^{-1}$, According to Table VI, our $J=0$, $E^{+}$levels up to $200 \mathrm{~cm}^{-1}$ are converged to within $0.01 \mathrm{~cm}^{-1}$. Convergence errors of other levels are probably similar or smaller. Full sets of $J=0$ and $J=1 E^{+}$and $E^{-}$levels are compared with their $[6+6] \mathrm{D}$ counterparts in SM Table IV. We find that the lowest $[6+6] \mathrm{D} E^{+}$level has the largest relative error. It is about $40 \%$ : [6+6] is $0.262 \mathrm{~cm}^{-1}$ and we get $0.405 \mathrm{~cm}^{-1}$. This error in the $[6+6] E^{+}$level will cause a significant error in the small bifurcation splitting that measures the shift of the average of the $A$ and $B$ prongs from the $E^{+}$energy. Column 3 
Publishipfg] able VI also shows that if we use only one MVW $\left(N_{v}=1\right)$, in the style of the calculation of Ref. 22, the energies relative to the ZPE have errors up to $1.26 \mathrm{~cm}^{-1}$, but the ZPE has a larger error of $11.60 \mathrm{~cm}^{-1}$.

Next, we compare triple fork origins and tunnelling splittings. They are reported in Table VIII. The tunnelling splittings are defined following previous papers[21, 26]. The interchange tunnelling splitting is defined as $i_{i}=\left|A_{i}^{ \pm}-B_{i}^{ \pm}\right|$. See Eq. 10 and Eq. 11 of Ref. 21 for the definition of the bifurcation tunnelling splitting (smallest) $b_{i}$. The acceptor switch (AS) tunnelling splitting (largest) is defined as by $a=o_{2}-o_{1}$. For a given triple fork, the $K=0$ origin $o_{i}(0)$ is defined as the average of the $A_{i}^{ \pm}$and $B_{i}^{ \pm} K=0$ energies for the fork. To determine origins for $K \neq 0, o_{i}(K), i=1,2$, we use [26]

$$
E_{i}(J, K)=o_{i}(K)+\frac{1}{2}(B+\widehat{C})\left[J(J+1)-K^{2}\right]
$$

where $E_{i}(J, K)$, with $i=1$ and 2 , is the average energy of the $A_{i}^{ \pm}(J, K)$ and $B_{i}^{ \pm}(J, K)$ levels. To get $o_{i}(1)$ from $J=K=1$ levels, one therefore needs $B+C$ for $K=1$, where $(B+C) / 2$ is an effective rotational constant. We could extract $B+C$, for $K=1$ from our calculated energies by taking half the difference between levels with $J=2$ and $J=1$. However, to do this we must assign a vibrational state label to each $J=2$ level and $K$ quantum numbers to each $J=2$ level. Instead, we simply take $B+C$ from the $[6+6] \mathrm{D}$ results. As our energy levels are very close to the $[6+6]$ D levels, the $B+C$ values must be close. This allows us to compute $o_{i}(1)$ using only $J=1$ levels. Interchange tunnelling splittings $i_{1}(K)$ and $i_{2}(K)$ are also given in Table VIII.

To get the origins and tunnelling splittings for each vibrational state, one also needs to assign vibrational states to levels. We simply use the assignments of Ref. 26. As an example, the raw levels and their assignments for $J=0$ are given in Table VII. Note that Leforestier et al[26] switched the assignments of OO and DT2 for $o_{2}$, from those of Ref. 21, based on the analysis of wavefunctions. Because OO and DT2 both have $A^{\prime}$ symmetry their wavefunctions may be strongly mixed and the assignment might not be meaningful. The changed assignment will, however, affect the AS splitting values. Of course, if coupling is so strong that that it is not possible to unambiguously associate $o_{2}$ and $o_{1}$ with vibrational states then the tunnelling splittings also become meaningless. Note that Leforestier's assignment switch means that although the DT2 $A_{1}^{+}$and $B_{1}^{+}$levels are higher than their OO counterparts the $\mathrm{OO} A_{2}^{-}$and $B_{2}^{-}$levels are higher than their DT2 counterparts. 
PublishingNot surprisingly, there is good agreement between our $12 \mathrm{D}$ and $[6+6] \mathrm{D}$ fork origins and tunnelling splittings (See Table VIII), consistent with the good agreement between the energy levels of the two calculations. At higher energies, $o_{1}(K=1)$ for $\mathrm{OO}$ of $[6+6] \mathrm{D}$ calculation is reported to be $153.54 \mathrm{~cm}^{-1}$, but we obtain $148.76 \mathrm{~cm}^{-1}$ from the data of Ref. 86 . This is probably due to the assignment uncertainty between OO and DT2 band.

The experimental AS tunnelling splitting $a(0)=11.18 \mathrm{~cm}^{-1}$, quoted in Ref. 26, is actually not an experimental value, but computed on the SAPT-5st surface[19]. Since all the experimental $\mathrm{O}_{2}$ values are given relative to the ground state-value of $o_{2}, 11.18 \mathrm{~cm}^{-1}$ is added to all experimental $o_{2}$ values, following Ref. 26. For the AS tunnelling splitting, only the sum $a(0)+a(1)$ has been measured experimentally. It is an important measure of the accuracy of the PES and bound state calculation methods. Table IX shows that our 12D number, 15.64 $\mathrm{cm}^{-1}$, is slightly closer to the experimental value $13.92 \mathrm{~cm}^{-1}$ than the $[6+6] \mathrm{D}$ value, 15.85 $\mathrm{cm}^{-1}$. Owing to the fact that our method includes no dynamical approximations, we can be certain that the o - c difference of $1.7 \mathrm{~cm}^{-1}$ means that the PES can be further improved.

For the small bifurcation splittings, there are experimental values for $b_{i}(0)+b_{i}(1)(i=1,2)$. Take $\left(b_{1}(0)+b_{1}(1)\right) / 2$ as an example. Table $\mathrm{X}$ shows that the $[6+6] \mathrm{D}$ value, $0.0862 \mathrm{~cm}^{-1}$, is almost 4 times larger than the experimental value, $0.0227 \mathrm{~cm}^{-1}$. Our $12 \mathrm{D}$ value, $0.0311 \mathrm{~cm}^{-1}$, is in much better agreement. This is simply because errors in the $E$ levels of the $[6+6] \mathrm{D}$ calculation are larger. They might be larger than those of the $A$ and $B$ levels because the density of the $E$ statés is larger. Perhaps surprisingly, the $6 \mathrm{D}$ result on the CCpol-8s PES gives the best agreement with experiment.

A semiclassical instanton method[24] has been used to compute tunnelling splittings of $\left(\mathrm{H}_{2} \mathrm{O}\right)_{2}$. Like our calculation, all coordinates are treated explicitly. The results for the AS splitting are poor. For the interchange and bifurcation splittings (the smallest) it seems to work rather well. [24] Unfortunately, these tiny splittings may depend on the PES and different PESs are use in our calculation and in Ref. 24.

\section{B. Water bend VRT levels}

There are two water bend vibrational states in $\left(\mathrm{H}_{2} \mathrm{O}\right)_{2}$ : the lower frequency acceptor bend and higher frequency donor bend. Each of these is split into 6 levels by tunnelling, 
Publishingthe same way as the ground state, and because these two states both transform like the $C_{s}$ irreducible representation $A^{\prime}$, the energy order of the irreducible representations is the same as in the ground state. The water bend VRT levels shown in Table XI are computed with Basis 1 and $N_{v}=10$. By comparing with a calculation done with Basis 2 and $N_{v}=$ 10 we infer that convergence errors are about $0.1 \mathrm{~cm}^{-1}$. It is one thing to compute levels in the water bend energy range. It is another to identify the water bend states. Due to the high density of states we need to use the wavefunctions (computed with ARPACK) to do this. For each wavefunction, $P_{v_{a}, v_{b}}=\sum_{L} c_{v_{a}, v_{b} ; L}^{2}$ is computed, where $c_{v_{a}, v_{b} ; L}$ is a component of an eigevector in the $\left|v_{a}, v_{b}\right\rangle|L\rangle$ basis of Eq. (6). Because the $\left|v_{a}\right\rangle$ and $\left|v_{b}\right\rangle$ we use are free $\mathrm{H}_{2} \mathrm{O}$ vibrational wavefunctions, $P_{10}=P_{01}$ are equal. For each symmetry, in most cases, we see two states with a large value of $P_{10}\left(\right.$ or $\left.P_{01}\right)$ and we simply assign the lower state as the acceptor bend and the higher state as the donor bend. Due to coupling, several of the acceptor bend states may also have significant $P_{10}$ or $P_{01}$ values. The energies of these additional states are very close (see Table XI) to the two with the largest $P_{10}$ or $P_{01}$ values. This coupling will further complicate the analysis of the spectrum.

Using the $[6+6] \mathrm{D}$ method, Leforestier et al. [26] calculated $J=0$ and $J=1$ water bend levels and published $R(0)$ and $P(1)$ lines. These lines can be directly compared with future rotationally resolved IR spectra. As a first step, we compute only $J=0$ water bend levels and compare them with $[6+6] \mathrm{D}$ levels $[26,86]$ in Table XI. To compute water bend levels with the $[6+6] \mathrm{D}$ approach, one must choose how to define the acceptor and donor adiabatic potentials. The $[6+6] \mathrm{D}$ levels we compare with are those obtained by assuming that the excitation energy migrates as the molecule moves from one equivalent minimum to another $\left(G_{16}\right.$ model $)$. There are large differences between the two sets of levels: our levels are about 4 and $11 \mathrm{~cm}^{-1}$ higher than [6+6]D levels for the acceptor and donor bends, respectively, even though there is good agreement for the ground state. The large difference between our water bend VRT leyels and their $[6+6] \mathrm{D}$ counterparts implies that the $[6+6] \mathrm{D}$ approximations are failing. It is certainly conceivable that the adiabatic approximation works less well for the excited states than for the ground state. Coupling between the adiabatic surfaces for the two (acceptor and donor) bends may be important and the non-adiabatic coupling is not included in $[6+6] \mathrm{D}$ calculations.

In Table XII, we compare the AS tunnelling splitting $a(0)$ and the interchange tunnelling 
Publishingitings $i_{1}$ and $i_{2}$ for the water bend states. These splittings for $[6+6] \mathrm{D}$ calculations are not discussed in Ref. 26, but we have computed them from the raw data sent to us. [86]. Our $i_{1}(K=0)$ and $i_{2}(K=0)$ for the acceptor bend are negative and those of the [6+6]D results are positive. This must be due to coupling with states of the same symmetry. Note, however, that in Ref. 26 it is clearly stated that $[6+6] \mathrm{D}$ is not good enough for the interchange tunnelling splittings. The AS tunnelling splitting $a(0)$ for the two calculations agree better, to within $0.2 \mathrm{~cm}^{-1}$. Our $a(0)$ for the acceptor and the donor are larger than that of the ground state.

There is only one gas phase experimental paper on the water bend states. [6] It, however, is not rotationally resolved. In Ref. 6 , the peak at $1600.6 \mathrm{~cm}^{-1}$ is assigned to an acceptor bend transition with $\Delta K=0$ and three peaks at $1613.8,1614.7$ and $1628.6 \mathrm{~cm}^{-1}$ to donor bend transitions with $\Delta K=1$. Assuming the $\Delta K=0$ assignment is correct and the peak at $1600.6 \mathrm{~cm}^{-1}$ corresponds to an $R(0), A_{1}^{+} \rightarrow A_{1}^{-}$transition, one can estimate the experimental $J=0, A_{1}$ level of the acceptor bend to be at $1600.6-\delta$ with $\delta=\left(B^{\prime}+C^{\prime}\right)+i_{1}^{\prime} \approx$ $1.2 \mathrm{~cm}^{-1}$ computed using the ground state values (see Fig. 1 of SM for an illustration of this equation). Using this single crude experimental number one might, with considerable uncertainty, conclude that the $[6+6] \mathrm{D} A_{1}^{+}$bend acceptor level is closer to the experiment than our 12D level. Of course, for a meaningful comparison between theory and experiment we must wait for a rotationally resolved experiment.

\section{CONCLUSION}

Using several computational methods, it is now possible to compute many numerically exact (ro-) vibrational energy levels and wavefunctions of molecules with 6 or more atoms. [26, 66, 68, 87-101] For molecules with three atoms such calculations are straightforward because it is possible to use a direct product basis composed of products of univariate functions. This simply strategy does not work for larger molecules because the size of the basis scales as $n^{3 N-6} \times(2 J+1)$, where $N$ is the number of atoms in the molecule, $n$ is the number of univariate functions per vibrational coordinate, and $J$ is the angular momentum quantum number. It is therefore necessary to use a better basis. Once one has chosen a basis it is necessary to evaluate Hamiltonian matrix-vector products (or in some cases calculate 
Publishihgrailtonian matrix elements). This is much easier when the PES is a sum of products $(\mathrm{SOP})[102]$ or in multimode $(\mathrm{MM})[103]$ form. The problem of computing a spectrum is much more difficult if the PES is not in SOP or MM form. In that case, one must use quadrature or collocation and deal with a large set of points. One is then confronted with the problem of needing to store values of the potential at the points

In this paper, we compute ro-vibrational levels of water dimer without storing the potential on a $12 \mathrm{D}$ grid. There are two key ideas. First, we use monomer vibrational wavefunctions as basis functions. [55] With only 10 6D MVWs we are able to compute converged levels in the $\mathrm{H}_{2} \mathrm{O}$ bend region of the spectrum of water dimer. If instead we used a basis of products of univariate functions of the 6 vibrational coordinates of the monomers we would need not 10 , but about $10^{6}$ basis functions! This reduction in basis size is important because the final basis is a (direct) product of the basis for the monomer vibrations, a basis for rotation of the dimer, and a basis for the 6 inter-monomer coordinates. The MVWs incorporate all of the coupling between the monomer vibrational coordinates. The final basis has product structure that is compatible with the structure of the KEO and therefore facilitates the calculation of KEO matrix elements. Second, to evaluate potential matrix-vector products we store an intermediate matrix, the $\boldsymbol{F}$ matrix. $[53,54]$ It has monomer vibrational labels and a label for a point on a grid for 6 the inter-monomer coordinates. Computing the $\boldsymbol{F}$ matrix is the most expensive part of the calculation, but its calculation is embarrassingly parallel.

Using these ideas we have calculated inter-molecular levels and many tunnelling splittings. They agree well with those reported previously by Leforestier and co-workers who used a $[6+6] \mathrm{D}$ method built on an adiabatic approximation, but to which other approximations are also added.[26] We also calculated frequency shifts for the acceptor and donor water bends. They agree poorly with those from the $[6+6] \mathrm{D}$ calculation. We have clearly demonstrated that using the ideas we introduce, it is not necessary to make dynamical approximations to compute ro-vibrational levels of water dimer. Nonetheless, it would be very costly to compute (and analyse) levels in the region of the $\mathrm{OH}$ stretches of $\mathrm{H}_{2} \mathrm{O}$. We used ARPACK to get as high as the the region of the water bend. The problem is the density of states. Methods designed to focus on a particular region of the spectrum that compute eigenvalues of a function $F(H)$ designed so that the spacing between the desired levels of $F(H)$ is increased might be useful. [104, 105] 


\section{PublishiKg. SUPPLEMENTARY MATERIAL}

In Supplementary Material (SM) we give $J=0,1,2$ energy levels from the $12 \mathrm{D}\left(\mathrm{H}_{2} \mathrm{O}\right)_{2}$ calculation (Table I, II, III). We also provide a detailed comparison of $E^{+}$and $E^{-}$levels of $\left(\mathrm{H}_{2} \mathrm{O}\right)_{2}$ that we have computed with those from the [6+6]D calculation (Table IV). In addition, the SM contains results of the $6 \mathrm{D}$ calculation: $\mathrm{J}=0,1,2$ levels (Table V, VI, VII) and a comparison of $6 \mathrm{D}$ fork origins and tunnelling splittings with those previous calculations (Table VIII). Finally, there is a diagram for understanding transitions to acceptor bend levels. (Fig. 1) 

FUNCTIONS FOR RIGID MONOMER

In this appendix, we start by presenting the effect of the $G_{16}$ symmetry operations on the uncoupled rigid monomer basis functions. The necessary symmetry operations are $E^{*}, P_{a b}$, (12) and (34). The uncoupled basis functions are

$$
\left\langle\mathbf{Q}, \alpha, \beta \mid \alpha_{0} ; j_{A}, k_{A}, m_{A} ; j_{B}, k_{B}, m_{B} ; J K\right\rangle
$$

The symmetry properties of the coupled basis functions have been discussed previously by Leforestier, van der Avoird and others.[18-20] See e.g. Table II of Ref. 18 and Table II of Ref. 19. We derive the symmetry properties of the uncoupled parity-adapted basis functions from those of the non parity-adapted functions, which are obtained by knowing the effect of the symmetry operations on the coordinates. We need to first resolve the ambiguity in the effect of the inversion operator $E^{*}$. There are-different ways to choose how $E^{*}$ effects to the coordinates we use. The most convenient choice for $\left(\mathrm{H}_{2} \mathrm{O}\right)_{2}$ is case II. See Appendix $\mathrm{B}$ of Ref. 55, where results were given in Table $\mathrm{V}$, for a detailed discussion. Having made this choice we can determine how $P_{a b},(12)$ and (34) affect the coordinates. We find the same results as those those given in Table 10 of Ref. 19, meaning these authors also use case II. From these relations, we find the effect of the symmetry operations on the non-parityadapted uncoupled basis which are listed here for the first time (omitting $\alpha_{0}$ which is not affected by any symmetry operations discussed here),

$$
\begin{aligned}
E^{*}\left|j_{A} k_{A} m_{A} ; j_{B} k_{B} m_{B} ; J K M\right\rangle & =(-1)^{J+k_{A}+k_{B}}\left|j_{A} \bar{k}_{A} \bar{m}_{A} ; j_{B} \bar{k}_{B} \bar{m}_{B} ; J \bar{K} M\right\rangle \\
P_{a b}\left|j_{A} k_{A} m_{A} ; j_{B} k_{B} m_{B} ; J K M\right\rangle & =(-1)^{J+j_{A}+j_{B}}\left|j_{B} \bar{k}_{B} \bar{m}_{B} ; j_{A} \bar{k}_{A} \bar{m}_{A} ; J \bar{K} M\right\rangle \\
(12)\left|j_{A} k_{A} m_{A} ; j_{B} k_{B} m_{B} ; J K M\right\rangle & =(-1)^{k_{A}}\left|j_{A} k_{A} m_{A} ; j_{B} k_{B} m_{B} ; J K M\right\rangle \\
(34)\left|j_{A} k_{A} m_{A} ; j_{B} k_{B} m_{B} ; J K M\right\rangle & =(-1)^{k_{B}}\left|j_{A} k_{A} m_{A} ; j_{B} k_{B} m_{B} ; J K M\right\rangle
\end{aligned}
$$

where $\bar{k}_{A} \equiv-k_{A}, \bar{m}_{A} \equiv-m_{A}$, and $\bar{K} \equiv-K$ etc. Combining Eqs. A2 to A5 and the $G_{16}$

group projection operators (Table I), one can derive $G_{16}$ symmetry-adapted uncoupled basis functions. They are different from their coupled basis counterparts[18, 19].

We prefer to work with parity-adapted uncoupled basis which has the advantage of being two times smaller. Using Eq. (A2), the parity adapted basis functions are built as linear 
Publishingr binations of two or one uncoupled basis functions,

$$
\begin{aligned}
& u_{j_{A} k_{A} m_{A} ; j_{B} k_{B} m_{B} ; K}^{J}=N_{m_{A} k_{A} k_{B} K} \frac{1}{\sqrt{2}} \\
& {\left[\left|j_{A} k_{A} m_{A} ; j_{B} k_{B} m_{B} ; J K M\right\rangle+(-1)^{J+k_{A}+k_{B}+P}\left|j_{A} \bar{k}_{A} \bar{m}_{A} ; j_{B} \bar{k}_{B} \bar{m}_{B} ; J \bar{K} M\right\rangle\right],}
\end{aligned}
$$

with

$$
N_{m_{A} k_{A} k_{B} K}=\left(1+\delta_{m_{A}, 0} \delta_{k_{A}, 0} \delta_{k_{B}, 0} \delta_{K, 0}\right)-1 / 2
$$

where $P=0$ and 1 correspond to even and odd parity, respectively. After setting $M=0$ and removing the normalization factor $1 / \sqrt{2 \pi}$ for $\alpha$, the parity-adapted basis functions are real for $(-1)^{J+P}=1$ and imaginary for $(-1)^{J+P}=-1$. They can be re-written,

$$
\begin{aligned}
& u_{j_{A} k_{A} m_{A} ; j_{B} k_{B} m_{B} ; K}^{J O}=\bar{d}_{m_{A} k_{A}}^{j_{A}}\left(\beta_{A}\right) \bar{d}_{m_{B} k_{B}}^{j_{B}}\left(\beta_{B}\right) \bar{d}_{0 K}^{J}(\beta) \\
\times & N_{m_{A} k_{A} k_{B} K}\left(\frac{1}{\sqrt{2 \pi}}\right)^{4} \sqrt{2} \cos \left(m_{A} \alpha_{A}+m_{B} \alpha_{B}+k_{A} \gamma_{A}+k_{B} \gamma_{B}\right), \text { for }(-1)^{J+P}=1 \\
& u_{j_{A} k_{A} m_{A} ; j_{B} k_{B} m_{B} ; K}^{J 0 P}=\bar{d}_{m_{A} k_{A}}^{j_{A}}\left(\beta_{A}\right) \bar{d}_{m_{B} k_{B}}^{j_{B}}\left(\beta_{B}\right) \bar{d}_{0 K}^{J}(\beta) \\
\times & \left(\frac{1}{\sqrt{2 \pi}}\right)^{4} \sqrt{2}(i) \sin \left(m_{A} \alpha_{A}+m_{B} \alpha_{B}+k_{A} \gamma_{A}+k_{B} \gamma_{B}\right), \text { for }(-1)^{J+P}=-1,
\end{aligned}
$$

where

$$
\bar{d}_{m_{A} k_{A}}^{j_{A}}=\sqrt{\frac{2 j_{A}+1}{2}} d_{m_{A} k_{A}}^{j_{A}}
$$

is a normalized Wigner $d$-function. [59] This explicit functional form for the parity-adapted functions is useful for plotting wavefunctions.

It is common to use rigid-monomer basis functions labelled by $j_{A B}$ obtained by using Clebsch-Gordan coefficients to add the angular momenta of monomers A and B, [26, 106, 107] but we prefer the uncoupled basis because in it potential matrix elements do not require Clebsch-Gordan cóefficients. The price we pay is that we have more non-zero matrix elements of the KEO.

For a single parity, the number of parity-adapted functions is roughly half the number of non-parity-adapted basis functions. The parity-adapted and non-parity-adapted bases span 
Publishithes same space if the quantum numbers are restricted by

$$
\begin{aligned}
& K \geq 0 \\
& m_{A} \geq 0 \quad(\text { if } K=0) \\
& k_{A} \geq 0 \quad\left(\text { if } m_{A}=K=0\right) \\
& k_{B} \geq 0 \quad\left(\text { if } k_{A}=m_{A}=K=0\right),
\end{aligned}
$$

where $\left|m_{X}\right| \leq m_{x},\left|k_{X}\right| \leq k_{x}$, and $|K| \leq J$ with $X=A, B$. Note that $m_{B}$ is not explicitly constrained because it depends on $K$ and $m_{A}$ via $K \equiv m_{A}+m_{B}$. These restrictions complicate the action of the symmetry operation on the parity-adapted functions because applying an operation may move a quantum number out of its allowed range.

To work with parity-adapted functions, we must know the effect of the four basic symmetry operations on the parity-adapted functions. Three of them are straightforward, the last one, $P_{a b}$, is a bit complicated because of the restrictions on the quantum numbers. The results are,

$$
\begin{aligned}
E^{*} u_{j_{A} k_{A} m_{A} ; j_{B} k_{B} m_{B} ; K}^{J M P} & =(-1)^{P} u_{j_{A} k_{A} m_{A} ; j_{B} k_{B} m_{B} ; K}^{J M P} \\
(12) u_{j_{A} k_{A} m_{A} ; j_{B} k_{B} m_{B} ; K}^{J M P} & =(-1)^{k} u_{j_{A} k_{A} m_{A} ; j_{B} k_{B} m_{B} ; K}^{J M P} \\
(34) u_{j_{A} k_{A} m_{A} ; j_{B} k_{B} m_{B} ; K}^{J M P} & =(-1)^{k_{B}} u_{j_{A} k_{A} m_{A} ; j_{B} k_{B} m_{B} ; K}^{J M P}
\end{aligned}
$$

and

$$
\begin{aligned}
P_{a b} u_{j_{A} k_{A} m_{A} ; j_{B} k_{B} m_{B} ; K}^{J M P}= & (-1)^{j_{A}+k_{A}+j_{B}+k_{B}+P} u_{j_{B} \bar{k}_{B} m_{B} ; j_{A} \bar{k}_{A} m_{A} ; K}^{J M P} \\
& \left(\text { if } K>0 \text { or } m_{A}=K=0 \text { and } k_{B}<0\right) \\
= & (-1)^{J+j_{A}+j_{B}} u_{j_{B} k_{B} \bar{m}_{B} ; j_{A} k_{A} \bar{m}_{A} ; 0}^{J M P} \\
& (\text { otherwise })
\end{aligned}
$$

\section{APPENDIX B: POTENTIAL INTEGRAL IN THE 6D PARITY-ADAPTED BA-}

\section{SIS}

If the two monomers are rigid, the potential depends on two polar angles $\left(\beta_{A}, \beta_{B}\right)$, three dihedral angles $\left(\alpha_{A}-\alpha_{B}, \gamma_{A}, \gamma_{B}\right)$ and $r_{0}$. The integration over the Euler angle $\beta$ (not to be confused with the $\beta$ that labels a $Q_{\beta}$ point) gives a unit factor since the potential does 
Publishingt depend on $\beta$. Moreover, the potential integral is diagonal in $J, P$, and $K$. A potential matrix element in the parity-adapted basis is (omitting the $r_{0}$ coordinate)

$$
\begin{aligned}
& \left\langle u_{j_{A}^{\prime} k_{A}^{\prime} m_{A}^{\prime} ; j_{B}^{\prime} k_{B}^{\prime} m_{B}^{\prime} ; K}^{J 0 P}|V| u_{j_{A} k_{A} m_{A} ; j_{B} k_{B} m_{B} ; K}^{J 0 P}\right\rangle \\
= & \int_{0}^{\pi} d \beta_{A} \sin \beta_{A} \bar{d}_{m_{A}^{\prime} k_{A}^{\prime}}^{j_{A}^{\prime}}\left(\beta_{A}\right) \bar{d}_{m_{A} k_{A}}^{j_{A}}\left(\beta_{A}\right) \int_{0}^{\pi} d \beta_{B} \sin \beta_{B} \bar{d}_{m_{B}^{\prime} k_{B}^{\prime}}^{j_{B}^{\prime}}\left(\beta_{B}\right) \bar{d}_{m_{B} k_{B}}^{j_{B}}\left(\beta_{B}\right) \\
\times & I_{m_{A}^{\prime} m_{B}^{\prime} k_{A}^{\prime} k_{B}^{\prime}, m_{A} m_{B} k_{A} k_{B}}\left(\beta_{A}, \beta_{B}\right)
\end{aligned}
$$

where $I$ is the integral over the dihedral angles. The integral over the two polar angles $\left(\beta_{A}, \beta_{B}\right)$ is evaluated by Gauss Legendre quadrature,

$$
\begin{aligned}
& \left\langle u_{j_{A}^{\prime} k_{A}^{\prime} m_{A}^{\prime} ; j_{B}^{\prime} k_{B}^{\prime} m_{B}^{\prime} ; K}^{J 0 P}|V| u_{j_{A} k_{A} m_{A} ; j_{B} k_{B} m_{B} ; K}^{J 0 P}\right\rangle \\
= & \sum_{p_{1}, p_{2}} T_{j_{A}^{\prime}, p_{1}}^{m_{A}^{\prime} k_{A}^{\prime}} T_{j_{B}^{\prime}, p_{2}}^{m_{B}^{\prime} k_{B}^{\prime}} I_{m_{A}^{\prime} m_{B}^{\prime} m_{A}^{\prime} k_{A}^{\prime} k_{B}^{\prime}, m_{A} m_{B} k_{A} k_{B}}^{p_{1}, p_{2}} T_{j_{A}, p_{1}}^{m_{A} k_{A}} T_{j_{B}, p_{2}}^{m_{B} k_{B}},
\end{aligned}
$$

where

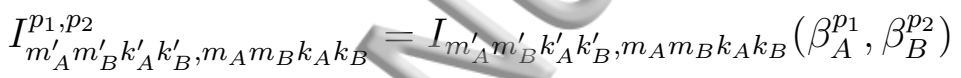

and

$$
\begin{aligned}
& T_{j_{A}, p_{1}}^{m_{A} k_{A}}=\sqrt{w_{p_{1}}} \bar{d}_{m_{A} k_{A}}^{j_{A}}\left(\beta_{A}^{p_{1}}\right) \\
& T_{j_{B}, p_{2}}^{m_{B} k_{B}}=\sqrt{w_{p_{2}}} \bar{d}_{m_{B} k_{B}}^{j_{B}}\left(\beta_{B}^{p_{2}}\right),
\end{aligned}
$$

with $\beta_{A}^{p_{1}}$ and $\beta_{B}^{p_{2}}$ being Gauss Legendre quadrature points, and $w_{p_{1}}$ and $w_{p_{2}}$ being the quadrature weights. We now fócus on the dihedral part of the potential integral, $I$, in Eq. (B1). Evaluating it by quadrature is not as simple because the factor in the parity-adapted functions (Eqs. A7 and A8) that depends on $\alpha_{A}, \alpha_{B}, \gamma_{A}$, and $\gamma_{B}$ is i) not a simple product and ii) depends on $(-1)^{J+P}$ and $K$. Since the potential depends only on the difference between $\alpha_{A}$ and $\alpha_{B}$, the integral over 4 dihedral angles can be reduced to an integral over 3 by the following variable substitution,

$$
\begin{aligned}
& \gamma=\alpha_{B} \\
& \tilde{\alpha}=\alpha_{A}-\alpha_{B} .
\end{aligned}
$$

In terms of the new coordinates after substituting $m_{B}=K-m_{A}$,

$$
m_{A} \alpha_{A}+m_{B} \alpha_{B}=K \gamma+m_{A} \tilde{\alpha}
$$


Publishihgring made this substitution, the integral over the dihedral angles no longer depends on $m_{B}$, but does depend on $K$, in which the potential is diagonal. This is similar to the transformation between coordinates used with a 2-angle embedded frame and coordinates used with a standard 3-angle embedded frame for methane; see Eqs. (106) and (107) in Appendix $\mathrm{B}$ of the EPAPS of Ref. 71. It can be proved that for any function $f\left(\alpha_{A}, \alpha_{B}\right)$ which is unchanged by shifting $\alpha_{A}$ or $\alpha_{B}$ by $2 \pi$

$$
\int_{0}^{2 \pi} d \alpha_{A} \int_{0}^{2 \pi} d \alpha_{B} f\left(\alpha_{A}, \alpha_{B}\right)=\int_{0}^{2 \pi} d \gamma \int_{0}^{2 \pi} d \tilde{\alpha} f\left(\alpha_{A}(\gamma, \tilde{\alpha}), \alpha_{B}(\gamma, \tilde{\alpha})\right) .
$$

Using this property, we find that the dihedral integrals fall into three cases:

case (a), $K=0$ and $(-1)^{J+P}=1$,

$$
\begin{aligned}
& I_{m_{A}^{\prime} m_{B}^{\prime} k_{A}^{\prime} k_{B}^{\prime}, m_{A} m_{B} k_{A} k_{B}}^{p_{1} p_{2}}=2\left(\frac{1}{2 \pi}\right)^{3} N_{m_{A}^{\prime} k_{A}^{\prime} k_{B}^{\prime} 0}^{\prime} N_{m_{A} k_{A} k_{B} 0} \int_{0}^{2 \pi} d \tilde{\alpha} \int_{0}^{2 \pi} d \gamma_{A} \int_{0}^{2 \pi} d \gamma_{B} \\
\times & \cos \left(m_{A}^{\prime} \tilde{\alpha}+k_{A}^{\prime} \gamma_{A}+k_{B}^{\prime} \gamma_{B}\right) V\left(\beta_{A}^{p_{1}}, \beta_{B}^{p_{2}}, \tilde{\alpha}, \gamma_{A}, \gamma_{B}\right) \cos \left(m_{A} \tilde{\alpha}+k_{A} \gamma_{A}+k_{B} \gamma_{B}\right)
\end{aligned}
$$

case (b), $K=0$ and $(-1)^{J+P}=-1$,

$$
\begin{aligned}
& I_{m_{A}^{\prime} m_{B}^{\prime} k_{A}^{\prime} k_{B}^{\prime}, m_{A} m_{B} k_{A} k_{B}}^{p_{2}}=2\left(\frac{1}{2 \pi}\right)^{3} \int_{0}^{2 \pi} d \tilde{\alpha} \int_{0}^{2 \pi} d \gamma_{A} \int_{0}^{2 \pi} d \gamma_{B} \\
\times & \sin \left(m_{A}^{\prime} \tilde{\alpha}+k_{A}^{\prime} \gamma_{A} 4 k_{B}^{\prime} \gamma_{B}\right) V\left(\beta_{A}^{p_{1}}, \beta_{B}^{p_{2}}, \tilde{\alpha}, \gamma_{A}, \gamma_{B}\right) \sin \left(m_{A} \tilde{\alpha}+k_{A} \gamma_{A}+k_{B} \gamma_{B}\right)
\end{aligned}
$$

case (c), $K>0$ and $(-1)^{J+P}=1$ or -1 ,

$$
\begin{aligned}
& I_{m_{A}^{\prime} m_{B}^{\prime} k_{A}^{\prime} k_{B}^{\prime}, m_{A} m_{B} k_{A} k_{B}}^{p_{1} p_{2}}=\left(\frac{1}{2 \pi}\right)^{3} \int_{0}^{2 \pi} d \tilde{\alpha} \int_{0}^{2 \pi} d \gamma_{A} \int_{0}^{2 \pi} d \gamma_{B} \\
\times & \cos \left[\left(m_{A}^{\prime}-m_{A}\right) \tilde{\alpha}+\left(k_{A}^{\prime}-k_{A}\right) \gamma_{A}+\left(k_{B}^{\prime}-k_{B}\right) \gamma_{B}\right] V\left(\beta_{A}^{p_{1}}, \beta_{B}^{p_{2}}, \tilde{\alpha}, \gamma_{A}, \gamma_{B}\right)
\end{aligned}
$$

Eqs. B8, B9, and B10 are similar to Eqs. (41), (42), and (43) of Ref. 71 for methane except that water dimer has three dihedral angles whereas methane has two.

In a later paper[72], we demonstrate, for methane, that matrix-vector products for the dihedral angle part are more efficiently evaluated using an FFT than by explicitly summing over basis and quadrature grid labels. Here we present equations, similar to those of Ref. 72, that show how to use FFTs for cases (b), (a) and (c). Consider first case (b). Eq. (B9) is similar to Eq. 13 of Ref. 72. As in Eq. 20 of Ref. 72, the potential matrix-vector product is computed by doing a real-to-complex FFT and a complex-to-real inverse FFT (dropping 
Publishíng, $\left.p_{2}\right)$ on all the vectors for brevity)

$$
\begin{aligned}
\tilde{u}_{q_{1}, q_{2}, q_{3}} / i & =\sum_{m_{A}=-m_{x}}^{m_{x}} \sum_{k_{A}=-k_{x}}^{k_{x}} \sum_{k_{B}=-k_{x}}^{k_{x}} \exp \left[-i 2 \pi\left(\frac{m_{A} q_{1}}{n_{q_{1}}}+\frac{k_{A} q_{2}}{n_{q_{2}}}+\frac{k_{B} q_{3}}{n_{q_{3}}}\right)\right] u_{m_{A} k_{A} k_{B}} . \\
u_{m_{A}^{\prime} k_{A}^{\prime} k_{B}^{\prime}}^{\prime} & =\frac{1}{n_{q_{1}} n_{q_{2}} n_{q_{3}}} \sum_{q_{1}=0}^{n_{q_{1}}-1} \sum_{q_{2}=0}^{n_{q_{2}}-1} \sum_{q_{3}=0}^{n_{q_{3}}-1} \exp \left[i 2 \pi\left(\frac{m_{A}^{\prime} q_{1}}{n_{q_{1}}}+\frac{k_{A}^{\prime} q_{2}}{n_{q_{2}}}+\frac{k_{B}^{\prime} q_{3}}{n_{q_{3}}}\right)\right] V_{q_{1}, q_{2}, q_{3}} \tilde{u}_{q_{1}, q_{2}, q_{3}} / i .
\end{aligned}
$$

Here $\left(q_{1}, q_{2}, q_{3}\right)$ label Fourier points for $\left(\tilde{\alpha}, \gamma_{A}, \gamma_{B}\right) .\left(n_{q_{1}}, n_{q_{2}}, n_{q_{3}}\right)$ are the numbers of Fourier points for $\left(\tilde{\alpha}, \gamma_{A}, \gamma_{B}\right)$. $V_{q_{1}, q_{2}, q_{3}}$ is the potential $V\left(\beta_{A}^{p_{1}}, \beta_{B}^{p_{2}}, \tilde{\alpha}, \gamma_{A}, \gamma_{B}\right)$ evaluated at the $3 \mathrm{D}$ Fourier points. Note that to use Eq. (B11), one must extend the range of the indices by removing the constraint of Eq. (A10) on $m_{A}, k_{A}, k_{B}$ The vector $u_{m_{A} k_{A} k_{B}}$ in Eq. (B11) is obtained from $u_{\underline{m_{A} k_{A} k_{B}}}$ using the equation,

$$
u_{-m_{A},-k_{A},<k_{B}}=-u_{\underline{m_{A} k_{A} k_{B}}}
$$

where $m_{A} k_{A} k_{B}$ means the indices are constrained according to Eq. (A10). Because of the symmetry of Eq. (B12), the vector $\tilde{u}_{q_{1}, q_{2}, q_{3}}^{\prime} / i$ computed with the real-to-complex FFT is pure imaginary. In the complex-to-real FFT library, the range of first index $q_{1}$ is halved, which means one needs to store only potential values on half of the grid, related to the fact that due to parity symmetry only half the potential values are required.

Case (a) is similar to case (b). As in Eq. 22 of Ref. 72, the potential matrix-vector product is computed by doing a real-to-complex FFT and a complex-to-real inverse FFT,

$$
\begin{gathered}
\tilde{u}_{q_{1}, q_{2}, q_{3}}=\sum_{m_{A}=-m_{x}}^{m_{x}} \sum_{k_{A}=-k_{x}}^{k_{x}} \sum_{k_{B}=-k_{x}}^{k_{x}} \exp \left[-i 2 \pi\left(\frac{m_{A} q_{1}}{n_{q_{1}}}+\frac{k_{A} q_{2}}{n_{q_{2}}}+\frac{k_{B} q_{3}}{n_{q_{3}}}\right)\right] u_{m_{A} k_{A} k_{B}} N_{m_{A} k_{A} k_{B} 0}^{-1} . \\
u_{m_{A}^{\prime} k_{A}^{\prime} k_{B}^{\prime}}=N_{m_{A}^{\prime} k_{A}^{\prime} k_{B}^{\prime} 0} \frac{1}{n_{q_{1}} n_{q_{2}} n_{q_{3}}} \sum_{q_{1}=0}^{n_{q_{1}}-1} \sum_{q_{2}=0}^{n_{q_{2}}-1} \sum_{q_{3}=0}^{n_{q_{3}}-1} \exp \left[i 2 \pi\left(\frac{m_{A}^{\prime} q_{1}}{n_{q_{1}}}+\frac{k_{A}^{\prime} q_{2}}{n_{q_{2}}}+\frac{k_{B}^{\prime} q_{3}}{n_{q_{3}}}\right)\right] V_{q_{1}, q_{2}, q_{3}} \tilde{u}_{q_{1}, q_{2}, q_{3}},
\end{gathered}
$$

where in input vector is obtained by extending the range of the constrained vector $u_{\underline{m_{A} k_{A} k_{B}}}$ using the equation,

$$
u_{-m_{A},-k_{A},-k_{B}}=u_{\underline{m_{A} k_{A} k_{B}}} .
$$

For case (c), we use the fact that Eq. (B10) is similar to Eq. 23 of Ref. 72. As in Eq. 24 of Ref. 72 , the potential matrix-vector product is computed by doing a complex-to-complex 
Publishifg and a complex-to-complex inverse FFT

$$
\begin{aligned}
\tilde{u}_{q_{1}, q_{2}, q_{3}} & =\sum_{m_{A}=-m_{x}}^{m_{x}} \sum_{k_{A}=-k_{x}}^{k_{x}} \sum_{k_{B}=-k_{x}}^{k_{x}} \exp \left[-i 2 \pi\left(\frac{m_{A} q_{1}}{n_{q_{1}}}+\frac{k_{A} q_{2}}{n_{q_{2}}}+\frac{k_{B} q_{3}}{n_{q_{3}}}\right)\right] u_{m_{A} k_{A} k_{B}} \\
u_{m_{A}^{\prime} k_{A}^{\prime} k_{B}^{\prime}}^{\prime} & =\frac{1}{n_{q_{1}} n_{q_{2}} n_{q_{3}}} \sum_{q_{1}=0}^{n_{q_{1}}-1} \sum_{q_{2}=0}^{n_{q_{2}}-1} \sum_{q_{3}=0}^{n_{q_{3}}-1} \exp \left[i 2 \pi\left(\frac{m_{A}^{\prime} q_{1}}{n_{q_{1}}}+\frac{k_{A}^{\prime} q_{2}}{n_{q_{2}}}+\frac{k_{B}^{\prime} q_{3}}{n_{q_{3}}}\right)\right] V_{q_{1}, q_{2}, q_{3}} \tilde{u}_{q_{1}, q_{2}, q_{3}}
\end{aligned}
$$

Note that there is no constraint on $\left(m_{A}, k_{A}, k_{B}\right)$ for $K>0$ and so there is no need to extend the vector before applying the FFT. $\tilde{u}_{q_{1}, q_{2}, q_{3}}^{\prime}$ is complex and the first index of its range is halved.

\section{ACKNOWLEDGMENTS}

This work has been supported by the Natural Sciences and Engineering Research Council of Canada. We thank Professor Leforestier for sending the unpublished energy levels of his calculations to us[86]. TC thanks the Alexander von Humboldt Society for support and the Manthe group for hospitality.

[1] T.R. Dyke, K.M.Mack, J. S., Muenter, J. Chem. Phys. 66, 498 (1997)

[2] E.N. Karyakin, G.T. Fraser, F. J. Lovas, R.D. Suenram, M. J. Fujitake, J. Chem. Phys. 102, $1114(1995)$

[3] K.L. Busarow, R.C. Cohen, G.A. Blake, K. B. Laughlin, Y. T. Lee, R. J. Saykally, J. Chem. Phys. 90, 3937 (1989)

[4] L.B. Braly, K. Liu, M. G. Brown, F. N. Keutsch, R. S. Fellers, R.J. Saykally, J. Chem. Phys. $112,10314(2000)$

[5] N. Pugliano, J. D. Cruzan, J. G. Loeser, R. J. Saykally, J. Chem. Phys. 98, 6600 (1993)

[6] J. B. Paul, R. A. Provencal, C. Chapo, K. Roth, R. Casaes, and R. J. Saykally, J. Phys. Chem. A, 103, 2972 (1999)

[7] F. N. Keutsch, N. Goldman, H. A. Harker, C. Leforestier, and R. J. Saykally Mol. Phys. 101, $3477(2003)$ 
H. A. Harker, F. N. Keutsch, C. Leforestier, Y. Scribano, J.-X. Han and R. J. Saykally Mol. Phys. 105, 497 (2007)

[9] H. A. Harker, F. N. Keutsch, C. Leforestier, Y. Scribano, J.-X. Han and R. J. Saykally Mol. Phys. 105, 513 (2007)

[10] B. E. Rocher-Casterline, L. C. Ch'ng, . K. Mollner, and H. Reisler, J. Chem. Phys. 134, $211101(2011)$

[11] W. T. S. Cole, R. S. Fellers, M. R. Viant, C. Leforestier, and R. J. Saykally J. Chem. Phys., 143, $154306(2015)$.

[12] A. Mukhopadhyay, W. T.S. Cole, and R. J. Saykally, Chem. Phys. Lett. 633, 13 (2015).

[13] T. R. Dyke, J. Chem. Phys. 66, 492 (1977)

[14] S. C. Althorpe and D. C. Clary J. Chem. Phys. 101, 3603 (1994).

[15] S. C. Althorpe and D. C. Clary J. Chem. Phys. 102, 4390 (1995).

[16] Jonathon K. Gregory and David C. Clary J. Chem. Phys. 102, 7817 (1995).

[17] H. Chen, S. Liu, and J. C. Light, J. Chem. Phys. 110, 168 (1999).

[18] C. Leforestier, L. B. Braly, K. Liu, M. J. Elroy, and R. J. Saykally, J. Chem. Phys. 106, 8527 (1997)

[19] G. C. Groenenboom, P. E. S. Wormer, A. van der Avoird, E. M. Mas, R. Bukowski, and K. Szalewicz, J. Chem. Phys. 113, 6702 (2000)

[20] M. J. Smit, G. C. Groenenboom, P. E. S. Wormer, and A. van der Avoird, R. Bukowski, and K. Szalewicz, J. Phys. Chem. 105, 6212 (2001).

[21] W. Cencek, K. Szalewicz, C. Leforestier, R. van Harrevelt, and A. van der Avoird, Phys. Chem. Chem. Phys. 10, 4716 (2008).

[22] R. E. A. Kelly, J. Tennyson, G. C. Groenenboom, and A. van der Avoird, J. Quant. Spectrosc. Radiat. Transfer. 111, 1262 (2010).

[23] J. Tennyson, M. J. Barber, and R. E. A. Kelly, Phil. Trans. R. Soc. A 370, 2656 (2012).

[24] J. O. Richardson, S. C. Althorpe, and D. J. Wales J. Chem. Phys. 135, 124109 (2011)

[25] C. Leforestier, F. Gatti, Raymond S. Fellers, and Richard J. Saykally J. Chem. Phys. 117, 8710 (2002).

[26] C. Leforestier, K. Szalewicz, and A. van der Avoid, J. Chem. Phys. 137, 014305 (2012).

[27] C. Leforestier, Phil. Trans. R. Soc. A 3702675 (2012) 
Publishin[g్g] A. Shank, Y. Wang, A. Kaledin, B. Braams, and J. M. Bowman, J. Chem. Phys. 130, 144314 (2009)

[29] Y. Wang, X. Huang, B. C. Shepler, B. J. Braams, and J. M. Bowman J. Chem. Phys. 134, $094509(2011)$

[30] V. Babin, C. Leforestier, and F. Paesani, J. Chem. Theo. Comp. 9, 5395 (2013).

[31] P. Jankowski, G. Murdachaew, R. Bukowski, O. Akin-Ojo, C. Leforestier, and K. Szalewicz J. Phys. Chem. A 119, 2940 (2015).

[32] H. A. Gebbie, W. J. Burroughs, J. Chamberlain, J. E. Harries, and R. G. Jones, Nature (London) 221, 143 (1969).

[33] U. Gora, R. Podeszwa, W. Cencek, and K. Szalewicz, J. Chem. Phys. 135, 224102 (2011).

[34] Volodymyr Babin, Gregory R. Medders, and Francesco Paesani J. Chem. Theory Comput., 10, 1599 (2014)

[35] T. Carrington, Jr., J. Chem. Phys. 146, 120902 (2017)

[36] Z. Bačić and J. C. Light, Annu. Rev. Phys. Chem. 40, 469 (1989).

[37] J. Tennyson, Comp. Phys. Rep. 4, 1 (1986).

[38] J. Bowman, T. Carrington, H.-D. Meyer, Mol. Phys. 106, 2145 (2008).

[39] G. Brocks, A. Van Der Avoird, B. T. Sutcliffe, and J. Tennyson, Mol. Phys. 50, 1025 (1983).

[40] C. Iung and C. Leforestier, J. Chem. Phys. 90, 3198 (1989) .

[41] M. J. Bramley and T. Carrington Jr, J. Chem. Phys. 99, 8519 (1993).

[42] R. Chen and H. Guo, J. Chem. Phys. 108, 6068 (1998).

[43] E. H. T. Olthof, A. van der Avoird, and P. E. S. Wormer, J. Chem. Phys. 101, 8430 (1994)

[44] E. H. T. Olthof, A. van der Avoird, and P. E. S. Wormer, J. G. Loeser, and R. J. Saykally J. Chem. Phys. 101, 8443 (1994)

[45] X.-G. Wang and T. Carrington, Jr., J. Chem. Phys. 113, 044313 (2000).

[46] A. van der Avoird and D. J. Nesbitt, J. Chem. Phys. 134, 044314 (2011).

[47] X.-G. Wang and T. Carrington, Jr., J. Chem. Phys. 143, 024303 (2015).

[48] X.-G. Wang, T. Carrington, Jr., J. Tang, and A. R. W. McKellar, J. Chem. Phys. 123, $034301(2005)$

[49] R. Dawes, X.-G. Wang, A. W. Jasper, and T. Carrington, Jr., J. Chem. Phys 133, 144306 (2010) 
X.-G. Wang, T. Carrington, Jr., R. Dawes, and A. W. Jasper, J. Mol. Spectrosc. 268, 53 (2011)

[51] J. Brown, X.-G. Wang, R. Dawes, and T. Carrington Journal of Chemical Physics 136134306 (2012)

[52] Ad van der Avoird, R. Podeszwa, K. Szalewicz, C. Leforestier, R. van Harrevelt, P. R. Bunker, M. Schnell, G. von Helden and G. Meijer, Phys. Chem. Chem. Phys. 12, 8219 (2010)

[53] X.-G. Wang and T. Carrington, Jr. J. Chem. Phys. 117 6923, (2002).

[54] H.-G. Yu, J. Chem. Phys. 117, 8190 (2002).

[55] X.-G. Wang and T. Carrington, Jr., J. Chem. Phys. 146, 104105 (2017)

[56] F. Gatti, J. Chem. Phys. 111, 7225 (1999).

[57] F.T. Smith, Phys. Rev. Lett. 45, 1157 (1980)

[58] X.-G. Wang and T. Carrington, Jr., Chem. Phys. Lett. 287, 289 (1998).

[59] R. N. Zare, Angular Momentum (Wiley: New York 1988).

[60] E. B. Wilson Jr., J. C. Decius, and P. C. Cross, Molecular Vibrations: The Theory of Infrared and Raman Vibrational Spectra (Dover, New York, 1980).

[61] X.-G. Wang and T. Carrington, Jr., J. Chem. Phys. 114, 1473(2001)

[62] R. Chen and H. Guo, J. Chem. Phys. 114, 1467 (2001)

[63] R. B. Lehoucq, D. C. Sorensen, and C. Yang, ARPACK Users Guide: Solu- tion of Large Eigenvalue Problems with Implicitly Restarted Arnoldi Meth- ods (SIAM, Philadelphia, 1998).

[64] X.-G. Wang and T. Carrington, Jr., J. Phys. Chem. A 111, 10220 (2007)

[65] J. F. Cornwell, Group Theory in Physics: An Introduction Academic, San Diego, CA, 1987, Appendix.

[66] H.-G. Yu, J/Chem. Phys. 120, 2270 (2004).

[67] X.-G. Wang and T. Carrington, Jr, J. Chem. Phys. 119, 101 (2003).

[68] X.-G. Wang and T. Carrington, Jr, J. Chem. Phys. 129, 234102 (2008).

[69] Hee-Seung Lee and John C. Light J. Chem. Phys., 120, 4626 (2004)

70] X.-G. Wang and T. Carrington, Jr, J. Chem. Phys. 118, 6946 (2003).

[71] X.-G. Wang and T. Carrington, Jr, J. Chem. Phys. 121, 2937 (2004).

[72] X.-G. Wang and T. Carrington, Jr, J. Chem. Phys. 123, 154303 (2005). 
Claude Leforestier The Journal of Chemical Physics 94, 6388 (1991)

[74] Claude Leforestier The Journal of Chemical Physics 101, 7357 (1994)

[75] Sarkar, P; Poulin, N; Carrington, T Journal of Chemical Physics 110, 10269-10274 (1999)

[76] Wang, Xiao-Gang; Carrington, Tucker, Journal of Chemical Physics Volume: 138104106 (2013)

[77] Xudong T. Wu and Edward F. Hayes The Journal of Chemical Physics 107, 2705 (1997)

[78] NITROGEN, Numerical and Iterative Techniques for Rovibronic Energies with General Internal Coordinates, a program by P. B. Changala, http://www.colorado.edu/nitrogen

[79] H. Wei and T. Carrington, Jr., J. Chem. Phys. 97, 3029 (1992).

[80] J. Echave and D. C. Clary, Chem. Phys. Lett. 190, 225 (1992).

[81] M.J. Bramley, J.W. Tromp, T. Carrington, and G.C. Corey, J. Chem. Phys. 100, 6175-6194 (1994)

[82] J. C. Light and T. Carrington Jr., Adv. Chem. Phys. 114, 263 (2000).

[83] J. C. Light, I. P. Hamilton, and J. V. Lill, J. Chem. Phys. 82, 1400 (1985)

[84] C. Leforestier, J. Chem. Phys. 94, 6388 (1991)

[85] L. H. Coudert and J. T. Hougen, J. Mol. Spectrosc. 130, 86 (1988).

[86] C. Leforestier, levels of $[6+6] \mathrm{D}$ calculations presented in Ref. 26, private communication, June 30th, 2017

[87] Gustavo. Avila and Tucker Carrington J. Chem. Phys. 137, 174108-1 - 174108-13 (2012)

[88] Gustavo. Avila and Tucker Carrington J. Chem. Phys. 134, 054126-1 054126-16 (2011)

[89] O. Vendrell, F. Gatti, D. Lauvergnat, and H.-D. Meyer, Angew. Chemie Int. Ed. 46, 6918 (2007).

[90] Thomas Halverson and Bill Poirier J. Phys. Chem. A, 119, 12417 (2015)

[91] S. Manzhos, T. Carrington Jr., L. Laverdure, N. Mosey, J. Phys. Chem. A 119, 9557 (2015)

[92] M.D. Coutinho-Neto, A. Viel, and U. Manthe J Chem Phys. 2004 Nov 15;121(19):9207-10.

[93] Phillip Thomas and Tucker Carrington J. Phys. Chem. A 119, 13074 (2015)

[94] Arnaud Leclerc and Tucker Carrington J. Chem. Phys. 140, 174111 (2014)

[95] James Brown and Tucker Carrington J. Chem. Phys.145, 144104 (2016)

[96] Joel M. Bowman , Stuart Carter and Xinchuan Huang International Reviews in Physical Chemistry, 22:3, 533-549, (2003) 
Ove Christiansen Phys. Chem. Chem. Phys., 2007, 9, 2942

[98] Guntram Rauhut The Journal of Chemical Physics 127, 184109 (2007)

[99] Marc Odunlami, Vincent Le Bris, Didier Bégué, Isabelle Baraille, and Olivier Coulaud The Journal of Chemical Physics 146, 214108 (2017)

[100] P. Felker and Z. Bačić The Journal of Chemical Physics 146, 084303 (2017)

[101] P. Felker and Z. Bačić Chemical Physics Letters 683, 172 (2017)

[102] B. E. Rocher-Casterline, L. C. Ch'ng, . K. Mollner, and H. Reisler, H. Romanowski, J. M. Bowman, and L. B. Harding, J. Chem. Phys. 82, 4155 (1985).

[103] S. Carter, S. J. Culik, and J. M. Bowman, J. Chem. Phys. 107, 10458 (1997).

[104] Hua-Gen Yu and Gunnar Nyman, J. Chem. Phys. 110, 11133 (1999)

[105] Shi-Wei Huang and Tucker Carrington J. Chem. Phys. 112, 8765-8771 (2000)

[106] Ad van der Avoird, Paul E. S. Wormer, and Robert Moszynski Chem. Rev. 94, 1931-1974 (1984)

[107] Dong H. Zhang, and John C. Light The Journal of Chemical Physics 104, 4544 (1996) 
\$13LE I: Definition of $G_{16}$ projection operators for $\left(\mathrm{H}_{2} \mathrm{O}\right)_{2}$. Our projection operator defintions are the same as those given in Table 9 of Ref. 20 except for $P^{E_{b}}$.

\begin{tabular}{ll}
\hline \hline Symmmetry $\Gamma$ & Projection operator $P^{\Gamma}$ \\
\hline$A_{1}^{+}$ & $(1 / 16)\left[E+E^{*}\right]\left[E+P_{a b}\right][E+(34)][E+(12)]$ \\
$B_{1}^{+}$ & $(1 / 16)\left[E+E^{*}\right]\left[E-P_{a b}\right][E+(34)][E+(12)]$ \\
$A_{2}^{+}$ & $(1 / 16)\left[E+E^{*}\right]\left[E-P_{a b}\right][E-(34)][E-(12)]$ \\
$B_{2}^{+}$ & $(1 / 16)\left[E+E^{*}\right]\left[E+P_{a b}\right][E-(34)][E-(12)]$ \\
$E_{a}^{+}$ & $(1 / 8)\left[E+E^{*}\right][E-(34)][E+(12)]$ \\
$E_{b}^{+}$ & $(1 / 8)\left[E+E^{*}\right][E+(34)][E-(12)]$ \\
& $(1 / 16)\left[E-E^{*}\right]\left[E+P_{a b}\right][E+(34)][E+(12)]$ \\
$A_{1}^{-}$ & $(1 / 16)\left[E-E^{*}\right]\left[E-P_{a b}\right][E+(34)][E+(12)]$ \\
$B_{1}^{-}$ & $(1 / 16)\left[E-E^{*}\right]\left[E-P_{a b}\right][E-(34)][E-(12)]$ \\
$A_{2}^{-}$ & $(1 / 16)\left[E-E^{*}\right]\left[E+P_{a b}\right][E-(34)][E-(12)]$ \\
$B_{2}^{-}$ & $(1 / 8)\left[E-E^{*}\right][E-(34)][E+(12)]$ \\
$E_{a}^{-}$ & $(1 / 8)\left[E-E^{*}\right][E+(34)][E-(12)]$ \\
$E_{b}^{-}$ &
\end{tabular}


PISLE II: $G_{8}$ symmetry-adapted basis functions for $\left(\mathrm{H}_{2} \mathrm{O}\right)_{2}$ obtained by applying $G_{4}$ projeciton operators to the parity-adapted basis functions defined in Eq. (6). The \pm label on the $G_{8}$ symmetry in the first column is consistent with $P$ in the third column. The superscripts $A$ and $B$ denote symmetric and anti-symmetric MVWs of $\mathrm{H}_{2} \mathrm{O}$ under $\mathrm{H}-\mathrm{H}$ permutation. The superscripts even and odd indicate the eveness and oddness of the quantum numbers $k_{A}$ and $k_{B}$.

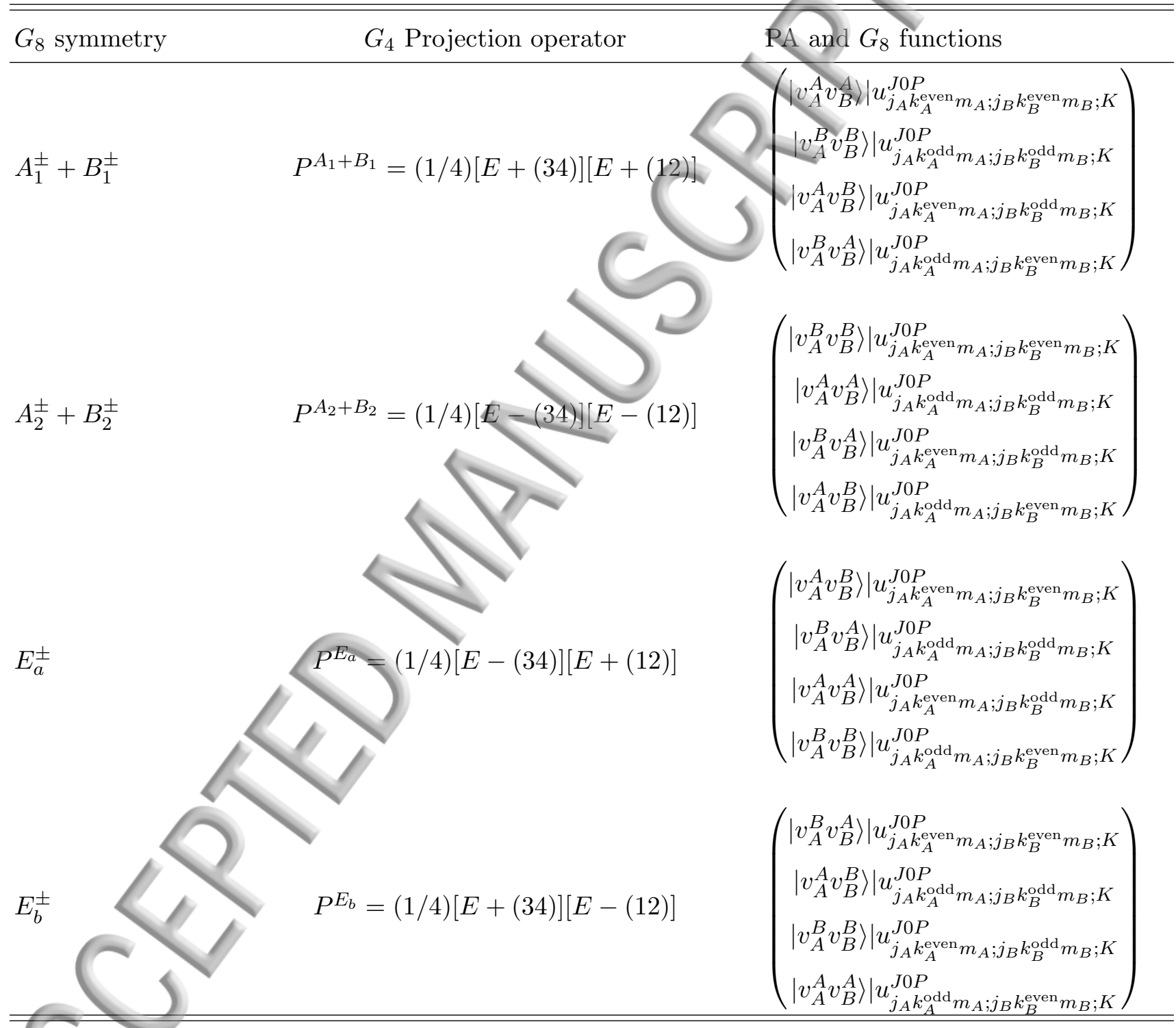


13LE III: Basis set paramters. $N_{r 0}$ is the number of tri-diagonal Morse DVR basis functions for stretch coordinate $r_{0} . j_{\max }$ is the maximum value of $\left(j_{X}, k_{X}, m_{X}\right)$ with $X=A$ and $B . N_{\theta}$ and $N_{\phi}$ are the numbers of Gauss-Legendre quadrature points for $\left(\beta_{A}, \beta_{B}\right)$ and Fourier points for $\left(\tilde{\alpha}, \gamma_{A}, \gamma_{B}\right) . N_{\text {bas }}$ is the number of even-parity basis functions. $N_{\mathbf{Q}}$ is the number of $\mathbf{Q}$ quadrature points retained under the ceiling value of $V_{\text {ceil }}\left(\right.$ in $\left.\mathrm{cm}^{-1}\right) . N_{\mathbf{q}}$ is the number of $\mathbf{q}$ DVR points for one monomer retained under the threshhold value of $V_{\text {cut }, \mathrm{A}}=29999 \mathrm{~cm}^{-1} \cdot 1 \mathrm{M}=1$ million.

\begin{tabular}{lccccccccc}
\hline \hline Basis & $N_{R_{1 X}} \times N_{R_{2 X}} \times N_{\theta_{X}}$ & $N_{\mathbf{q}}$ & $N_{r 0}$ & $j_{\max }$ & $N_{\theta}$ & $N_{\phi}$ & $N_{\text {bas }}$ & $N_{\mathbf{Q}}($ ratio $)$ & $V_{\text {ceil }}$ \\
\hline Basis 1 & $7 \times 7 \times 24$ & 803 & 7 & 12 & 13 & 26 & $1.387 \mathrm{M} \times N_{v}$ & $2.706 \mathrm{M}(83 \%)$ & 1000 \\
Basis 1f & $7 \times 7 \times 24$ & 803 & 7 & 12 & 13 & 26 & $1.387 \mathrm{M} \times N_{v}$ & $3.245 \mathrm{M}(100 \%)$ & 29999 \\
& & & & & & & & & \\
Basis 2 & $7 \times 7 \times 24$ & 803 & 6 & 10 & 11 & 22 & $0.516 \mathrm{M} \times N_{v}$ & $0.589 \mathrm{M}(47 \%)$ & 0 \\
\hline
\end{tabular}


TABLE IV: The effect of symmetry operations on the dihedral angles of water dimer.

\begin{tabular}{llll}
\hline \hline Operator & \multicolumn{3}{c}{ angles } \\
\hline$E$ & $\gamma_{B}$ & $\gamma_{A}$ & $\alpha_{A}-\alpha_{B}$ \\
$E^{*}$ & $-\gamma_{B}$ & $-\gamma_{A}$ & $-\left(\alpha_{A}-\alpha_{B}\right)$ \\
$(12)$ & $\gamma_{B}$ & $\gamma_{A}+\pi$ & $\alpha_{A}-\alpha_{B}$ \\
$(34)$ & $\gamma_{B}+\pi$ & $\gamma_{A}$ & $\left.\alpha_{A}-\alpha_{B}\right)$ \\
& & & $-\left(\alpha_{A}-\alpha_{B}\right)$ \\
$(34)^{*}$ & $\pi-\gamma_{B}$ & $-\gamma_{A}$ & $-\left(\alpha_{A}-\alpha_{B}\right)$ \\
$(12)(34)^{*}$ & $\pi-\gamma_{B}$ & $\pi-\gamma_{A}$ & \\
\hline \hline
\end{tabular}


\$13LE V: Optimization of $r_{0}$ basis functions: TDM-DVR basis is more efficient than PODVR basis. Shown are the $6 \mathrm{D} E^{+}$vibrational levels of $\left(\mathrm{H}_{2} \mathrm{O}\right)_{2}\left(\mathrm{in}^{-1}\right)$ computed on CCpol-8s[21] PES relative to the $\mathrm{ZPE}$. The first level is $\mathrm{ZPE}$ with $A_{1}^{+}$symmetry. The others are $E^{+}$levels.

\begin{tabular}{crrr}
\hline \hline & $\begin{array}{r}\text { Error } \\
\text { PODVR }\end{array}$ & $\begin{array}{r}\text { Error } \\
\text { TDM-DVR }\end{array}$ & $\begin{array}{r}\text { Benchmark } \\
\text { sine-DVR }\end{array}$ \\
$N_{r_{0}}$ & 9 & 7 & 120 \\
\hline ZPE & 0.001 & 0.000 & -1094.199 \\
$v=1$ & 0.000 & 0.000 & 0.433 \\
$v=2$ & 0.000 & 0.000 & 61.993 \\
$v=3$ & -0.001 & -0.003 & 109.272 \\
$v=4$ & -0.003 & -0.003 & 117.717 \\
$v=5$ & -0.002 & -0.004 & 130.040 \\
$v=6$ & 0.003 & -0.003 & 147.990 \\
$v=7$ & -0.020 & -0.020 & 172.301 \\
$v=8$ & -0.002 & -0.005 & 191.402 \\
$v=9$ & -0.002 & -0.004 & 207.152 \\
\hline \hline
\end{tabular}


DISE VI: Convergence tests for $\left(\mathrm{H}_{2} \mathrm{O}\right)_{2}$ levels. The first $9 E+$ vibrational levels are used to do the convergence tests. Levels are given relative to the zero point energy (ZPE). The levels in columns 2, 3, 4, 5 are also relative to those of column 6 (Basis 1f), except for the ZPE.

\begin{tabular}{lrrrrrr}
\hline \hline & Basis 2 & Basis 1 & Basis 1 & Basis 1 & Basis 1f & {$[6+6] \mathrm{D}$} \\
\hline$N_{v}$ & 10 & 1 & 10 & 48 & 48 & \\
ZPE & 8161.928 & 8171.354 & 8160.452 & 8159.758 & 8159.758 & \\
$D_{0}$ & 2.17 & 11.60 & 0.69 & 0.095 & -1109.744 & -1108.265 \\
$\mathrm{v}=1$, & 0.09 & -0.01 & 0.00 & 0.000 & 0.405 & 0.262 \\
$\mathrm{v}=2$, & -0.09 & 0.10 & 0.02 & 0.000 & 62.213 & 61.842 \\
$\mathrm{v}=3$, & -0.05 & 0.13 & 0.02 & -0.002 & 109.307 & 109.055 \\
$\mathrm{v}=4$, & -0.11 & 0.17 & 0.04 & -0.002 & 117.409 & 117.433 \\
$\mathrm{v}=5$ & -0.26 & 0.97 & 0.03 & -0.001 & 130.019 & 129.599 \\
$\mathrm{v}=6$ & 0.00 & -0.18 & 0.05 & 0.000 & 143.411 & 143.295 \\
$\mathrm{v}=7$ & -0.32 & 1.26 & 0.04 & -0.004 & 172.240 & 171.946 \\
$\mathrm{v}=8$ & -0.18 & 0.55 & 0.06 & -0.001 & 189.368 & 189.318 \\
$\mathrm{v}=9$ & -0.10 & 0.27 & 0.09 & -0.003 & 205.332 & \\
\hline \hline
\end{tabular}


\$13LE VII: $J=0 A / B$ levels (in $\left.\mathrm{cm}^{-1}\right)$ of $\left(\mathrm{H}_{2} \mathrm{O}\right)_{2}$ up to $200 \mathrm{~cm}^{-1}$ relative to the ZPE of 8160.4524 $\mathrm{cm}^{-1}$. They are used to compute the fork origins and tunnelling splittings of Table VIII. Levels are obtained with Basis 1 and $N_{v}=10$. Vibrational state labels are defined in the footnote of Table VIII.

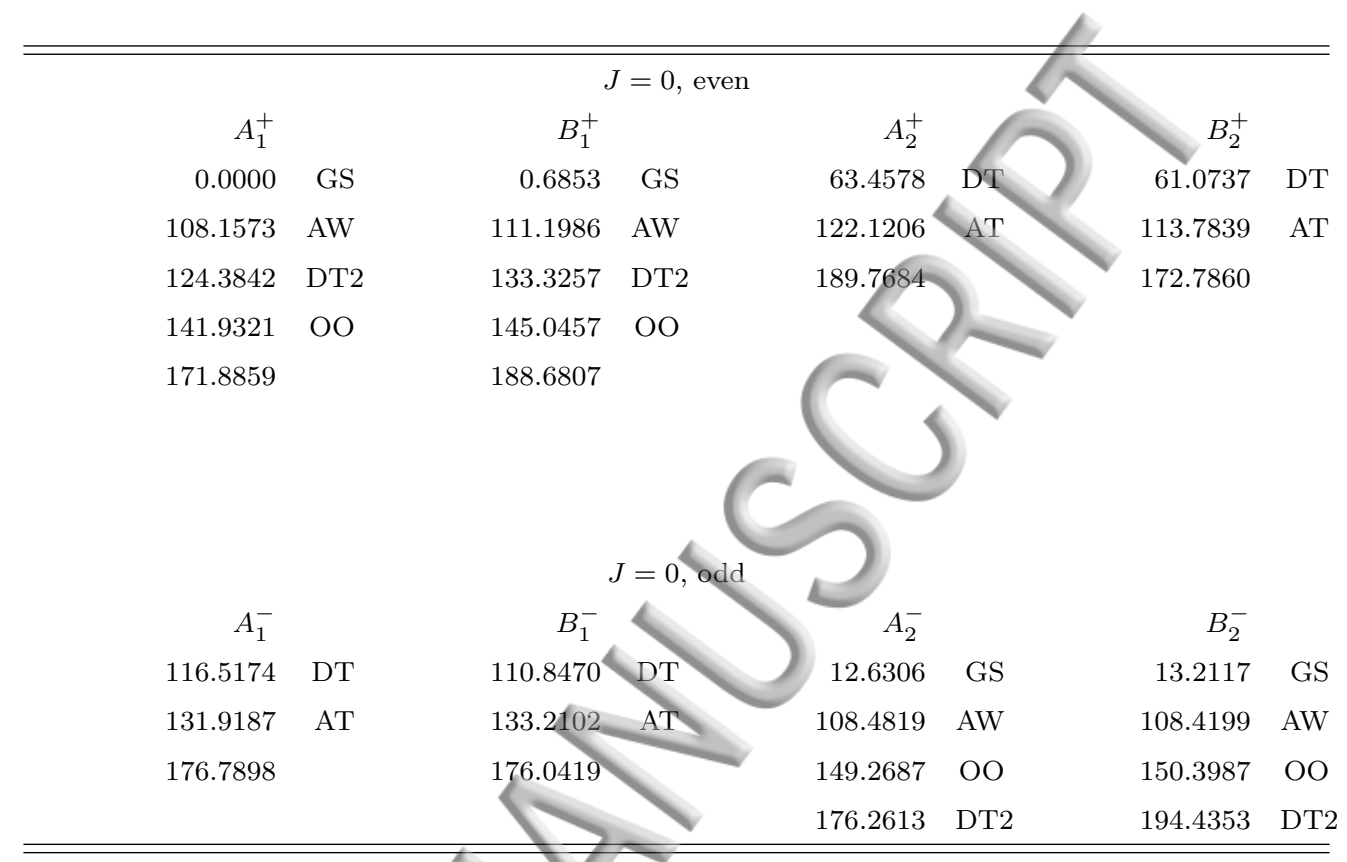


$\mathrm{OO}$ is the inter-molecular stretch state. The labels (1) and (2) correspond to the energies $\left(\mathrm{cm}^{-1}\right)$ of the origins $o_{1}$ and $o_{2}$. The numbers in parentheses are the interchange tunnelling splittings of the corresponding triplet $\left(i_{1}\right.$ and $\left.i_{2}\right)$.

\begin{tabular}{|c|c|c|c|c|c|c|c|c|}
\hline & & $12 \mathrm{D}$ & {$[6+6] \mathrm{D}$} & Expt & & $12 \mathrm{D}$ & {$[6+6] \mathrm{D}$} & Expt \\
\hline \multirow[t]{2}{*}{$\mathrm{OO}$} & (2) & $149.49(1.13)$ & $149.63(1.23)$ & $153.62(1.88)$ & (1) & $152.08(1.3$ & $152.07(1.48)$ & \\
\hline & (1) & $143.15(3.11)$ & $143.20(3.27)$ & & $(2)$ & & $53.54(2.54)^{a}$ & \\
\hline \multirow[t]{2}{*}{$\mathrm{DT}^{2}$} & (2) & $185.01(18.2)$ & $184.57(18.3)$ & & $(2)$ & & $173.73(19.0)^{b}$ & \\
\hline & (1) & $128.51(8.94)$ & $128.22(9.19)$ & & (1) & $162.14(9.29)$ & $161.61(9.10)^{b}$ & \\
\hline \multirow[t]{2}{*}{ AT } & (1) & $132.22(1.29)$ & $132.10(1.48)$ & & & & $142.42(4.04)$ & \\
\hline & (2) & $117.61(8.34)$ & $117.50(8.67)$ & $120.19(9.39)$ & 2) & $136.33(4.50)$ & $136.52(4.66)$ & \\
\hline \multirow[t]{2}{*}{ AW } & (2) & $108.11(0.06)$ & $107.82(0.10)$ & & (2) & $123.27(3.00)$ & $123.12(3.16)$ & $123.56(3.41)$ \\
\hline & (1) & $109.34(3.04)$ & 109.23(3.29) & & (1) & $108.49(4.58)$ & $108.28(4.76)$ & $109.98(5.24)$ \\
\hline \multirow[t]{2}{*}{ DT } & (1) & $113.34(5.67)$ & $113.35(5.91)$ & & (2) & $92.75(3.17)$ & $92.18(3.34)$ & \\
\hline & (2) & $61.92(2.38)$ & $61.33(2.4$ & & (1) & $86.49(1.28)$ & $86.37(1.32)$ & $87.75(1.11)$ \\
\hline \multirow[t]{3}{*}{ GS } & (2) & $12.58(0.58)$ & $12.75(0.61)$ & $18(0.65)^{c}$ & (1) & $15.35(0.63)$ & $15.45(0.67)$ & $14.39(0.70)$ \\
\hline & (1) & $0.00(0.69)$ & $0.00(0$. & & $(2)$ & $12.29(0.49)$ & $12.36(0.51)$ & $11.66(0.54)$ \\
\hline & & & & & & & $K=1$ & \\
\hline
\end{tabular}

The abbreviations and the point group $C_{s}$ symmetries are Ground State (GS, $A^{\prime}$ ), Donor Torsion (DT, $\left.A^{\prime \prime}\right)$, Acceptor Wag $\left(\mathrm{AW}, A^{\prime}\right)$, Acceptor Twist (AT, $\left.A^{\prime \prime}\right)$, inter-molecular stretch (OO, $\left.A^{\prime}\right)$, Donor Torsion overtone (DT2, $\left.A^{\prime}\right)$.

a 153.54 may be a typo. We obtain $148.76 \mathrm{~cm}^{-1}$ computed from data of Ref. 86 . ${ }^{b}$ Absent from Ref. 26 and computed by us from data of Ref. 86.

${ }^{c}$ The AS tunnelling splitting $\mathrm{a}(0)=11.18 \mathrm{~cm}^{-1}$ is actually not an experimental value, but computed on the SAPT-5st surface. 
TABLE IX: The AS tunnelling splittings $a(0)$ and $a(1)$ for the ground state.

\begin{tabular}{lrrrr}
\hline \hline & Expt $^{a}$ & This work & {$[6+6] \mathrm{D}^{b}$} & ${\text { CCpol-8s }(6 \mathrm{D})^{a}}^{a}$ \\
\hline$a(K=0)$ & $\mathrm{N} / \mathrm{A}$ & 12.58 & 12.75 & 12.39 \\
$a(K=1)$ & $\mathrm{N} / \mathrm{A}$ & 3.06 & 3.10 & 2.93 \\
$a(K=0)+a(K=1)$ & 13.92 & 15.64 & 15.85 & 15.32 \\
\hline \hline
\end{tabular}

${ }^{a}$ Ref. $7 .{ }^{b}$ Ref. 26. ${ }^{c}$ Ref. 21. 
This manuscript was accepted by J. Chem. Phys. Click here to see the version of record.

TABLE X: Bifurcation splittings $b_{1}(K)$ and $b_{2}(K)\left(\right.$ in $\left.\mathrm{cm}^{-1}\right)$.

\begin{tabular}{lrrrr}
\hline \hline & Expt. ${ }^{a}$ & This work & \multicolumn{2}{c}{$[6+6] \mathrm{D}^{b}$ CCpol-8s $(6 \mathrm{D})^{c}$} \\
\hline$b_{1}(0)$ & $\mathrm{N} / \mathrm{A}$ & 0.0330 & -0.0490 & 0.0218 \\
$b_{1}(1)$ & $\mathrm{N} / \mathrm{A}$ & -0.0293 & -0.1235 & -0.0198 \\
$\left(\left|b_{1}(0)\right|+\left|b_{1}(1)\right|\right) / 2$ & 0.0227 & 0.0311 & 0.0862 & 0.0208 \\
& & & & \\
$b_{2}(0)$ & $\mathrm{N} / \mathrm{A}$ & 0.0327 & 0.1198 & 0.0216 \\
$b_{2}(1)$ & $\mathrm{N} / \mathrm{A}$ & -0.0357 & 0.0521 & -0.0255 \\
$\left(\left|b_{2}(0)\right|+\left|b_{2}(1)\right|\right) / 2$ & 0.0249 & 0.0342 & 0.0859 & 0.0236 \\
\hline \hline
\end{tabular}

${ }^{a}$ Ref. $7{ }^{b}$ Computed by us from data of Ref. $86 .^{c}$ Ref. 21.

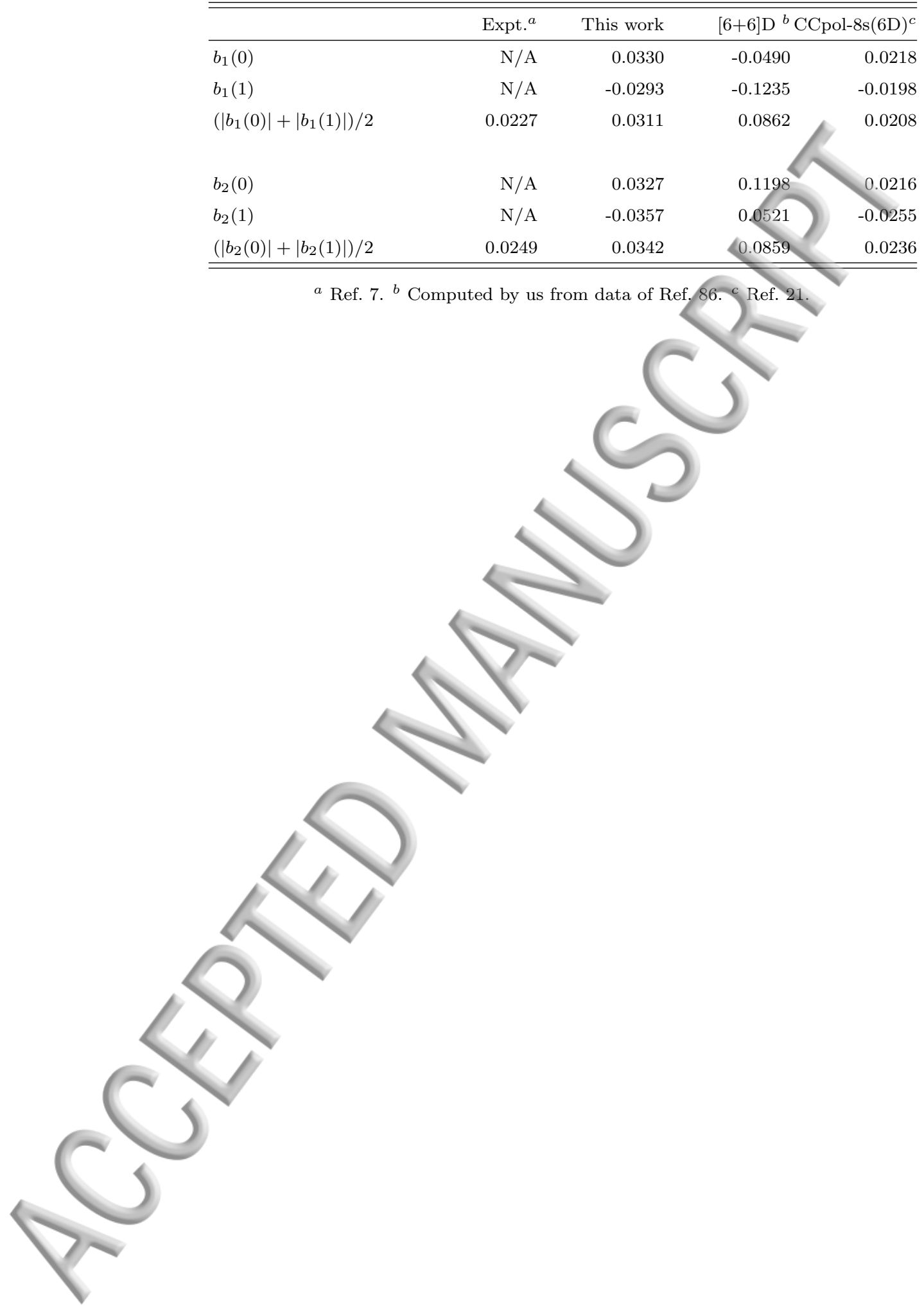


T13LE XI: $\mathrm{H}_{2} \mathrm{O}$ bending vibrational levels $\left(\right.$ in $\left.\mathrm{cm}^{-1}\right)$ of $\left(\mathrm{H}_{2} \mathrm{O}\right)_{2}$. The numbers in parentheses in column 3 are the contribution of $\left|v_{a} v_{b}\right\rangle=|10\rangle$ and $|01\rangle$ in the basis of Eq. (6): $P_{10}+P_{01}$. $E_{2}$ is the [6+6]D calculation of Ref. 26. $\Delta=E_{1}-E_{2}$.

\begin{tabular}{lllrr}
\hline \hline & & $E_{1}$ (This work) & $E_{2}[6+6] \mathrm{D}$ & $\Delta$ \\
\hline bend[Acceptor] & $A_{1}^{+}$ & $1605.09(0.54)$ & 1600.65 & 4.44 \\
& $B_{1}^{+}$ & $1604.55(0.36)^{a}$ & 1601.51 & 3.04 \\
& $A_{2}^{-}$ & $1618.47(0.50)^{b}$ & 1613.92 & 4.55 \\
& $B_{2}^{-}$ & $1618.02(0.58)$ & 1614.61 & 3.41 \\
bend[Donor] & & & & \\
& $A_{1}^{+}$ & $1617.74(0.66)$ & 1606.81 & 10.93 \\
& $B_{1}^{+}$ & $1618.18(0.84)$ & 1607.65 & 10.53 \\
& $A_{2}^{-}$ & $1631.79(0.78)$ & 1620.80 & 10.99 \\
& $B_{2}^{-}$ & $1632.13(0.86)$ & 1621.51 & 10.62 \\
\hline \hline
\end{tabular}

${ }^{a}$ Two other candidates: $1604.48(0.28)$ and $1604.65(0.26)$.

${ }^{b}$ Another candidate: 1618.26(0.38). 
\$13LE XII: The AS tunnelling splitting and interchange tunnelling splitting(in $\mathrm{cm}^{-1}$ ) for the ground state (GS) and two water bend states.

\begin{tabular}{lrr}
\hline \hline & This work & {$[6+6] \mathrm{D}^{a}$} \\
\hline$a(K=0)$ GS & 12.50 & 12.75 \\
$a(K=0)$ bend[Acceptor] & 13.42 & 13.19 \\
$a(K=0)$ bend[Donor] & 14.00 & 13.93 \\
& & \\
$i_{1}(K=0)$ GS & 0.69 & 0.72 \\
$i_{2}(K=0)$ GS & 0.58 & 0.61 \\
$i_{1}(K=0)$ bend[Acceptor] & -0.54 & 0.86 \\
$i_{2}(K=0)$ bend[Acceptor] & -0.45 & 0.69 \\
$i_{1}(K=0)$ bend[Donor] & 0.44 & 0.84 \\
$i_{2}(K=0)$ bend[Donor] & 0.34 & 0.71 \\
\hline \hline & \multicolumn{2}{c}{ Computed by us from data of Ref. 86.}
\end{tabular}




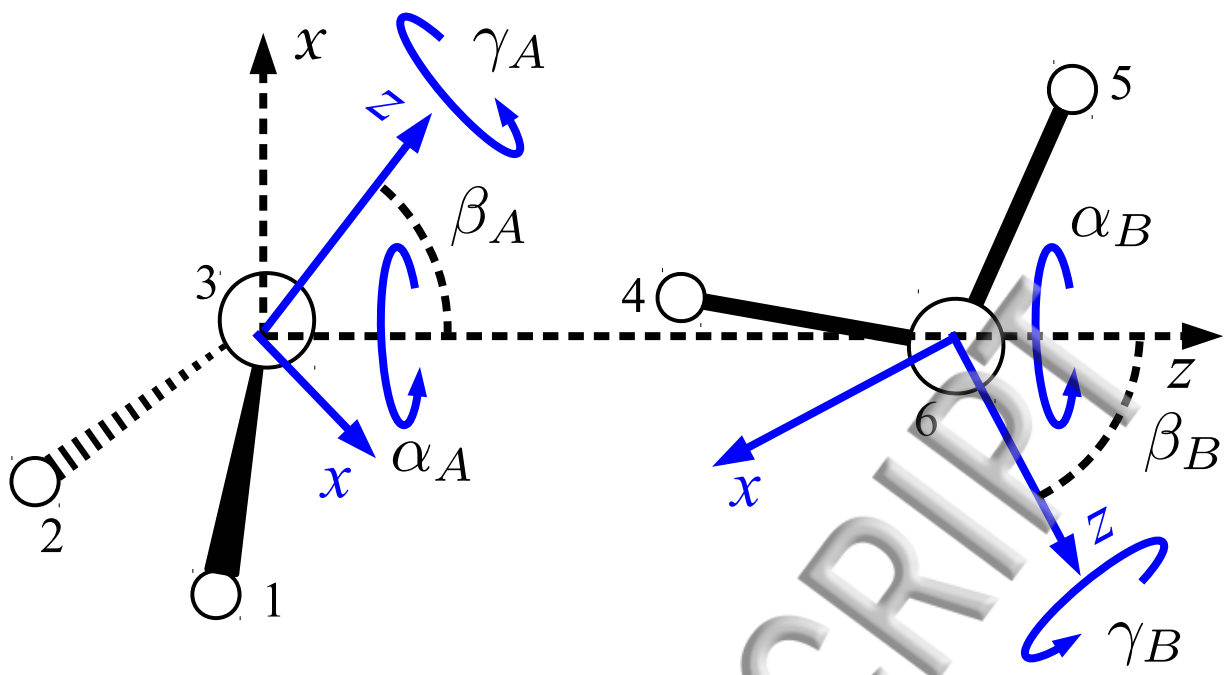

FIG. 1: The coordinates for $\left(\mathrm{H}_{2} \mathrm{O}\right)_{2}$. The DF frame-is indicatd by dashed $x-z$ axes. The two MF frames are indicatd by blue $x$ - $z$ axes. The MF frame is the bisector frame; the $z$-axis points away from the $\mathrm{H}$ atoms. and the $x$-axis is the on the $\mathrm{H}_{2} \mathrm{O}$ plane with $\mathrm{H}_{1}\left(\right.$ or $\left.\mathrm{H}_{4}\right)$ having a positive $x$ coordinate. With the acceptor at the left labelled as $A$ and donor as $B$, the coordinates at equilibrium are $\left(\alpha_{A}, \beta_{A}, \gamma_{A} ; \alpha_{B}, \beta_{B}, \gamma_{B}\right)=\left(0.0^{\circ}, 54.36^{\circ}, 90.0^{\circ} ; 180^{\circ}, 60.05^{\circ}, 0^{\circ}\right) . r_{0 e}=5.5088 a_{0}$ , $\left(R_{1 A}, R_{2 A}, \theta_{A}\right)=\left(1.7739 a_{0}, 1.7739 a_{0}, 107.93^{\circ}\right),\left(R_{1 B}, R_{2 B}, \theta_{B}\right)=\left(1.7792 a_{0}, 1.7712 a_{0}, 107.96^{\circ}\right)$. The minimum energy is $D_{e}=-1754.52 \mathrm{~cm}^{-1}$. If the donor is at the left (see e.g. Fig. 1 of Ref. 19), the angles are $\left(\alpha_{A}, \beta_{A}, \gamma_{A}, \alpha_{B}, \beta_{B}, \gamma_{B}\right)=\left(0.0^{\circ}, 119.95^{\circ}, 0.0^{\circ} ; 180^{\circ}, 125.64^{\circ}, 90.0^{\circ}\right)$. The point with the acceptor at the left and the point with acceptor at the right are linked by the symmetry operation $P_{a b}^{*}: P_{a b}^{*}\left(\alpha_{A}, \beta_{A}, \gamma_{A} ; \alpha_{B}, \beta_{B}, \gamma_{B}\right)=\left(\pi+\alpha_{B}, \pi-\beta_{B}, \pi-\gamma_{B} ; \pi+\alpha_{A}, \pi-\beta_{A}, \pi-\gamma_{A}\right)$. 


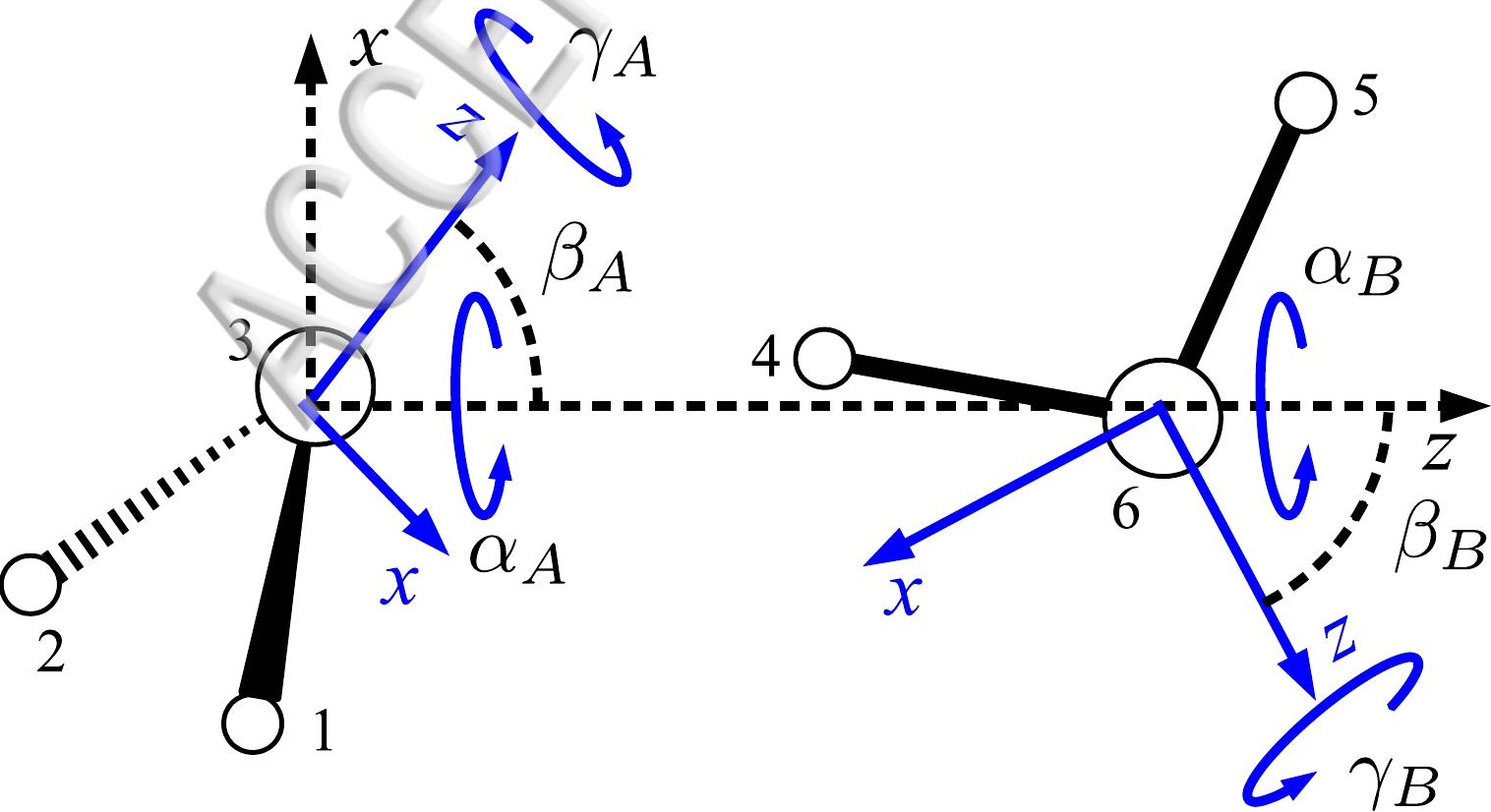

